\title{
Mass renormalization in string theory: general states
}

\author{
Roji Pius, ${ }^{a}$ Arnab Rudra ${ }^{b}$ and Ashoke Sen ${ }^{a}$ \\ ${ }^{a}$ Harish-Chandra Research Institute, \\ Chhatnag Road, Jhusi, Allahabad 211019, India \\ ${ }^{b}$ Department of Applied Mathematics and Theoretical Physics, \\ Wilberforce Road, Cambridge CB3 OWA, U.K. \\ E-mail: rojipius@mri.ernet.in, A.Rudra@damtp.cam.ac.uk, \\ sen@mri.ernet.in
}

ABSTRACT: In a previous paper we described a procedure for computing the renormalized masses and S-matrix elements in bosonic string theory for a special class of massive states which do not mix with unphysical states under renormalization. In this paper we extend this result to general states in bosonic string theory, and argue that only the squares of renormalized physical masses appear as the locations of the poles of the S-matrix of other physical states. We also discuss generalizations to Neveu-Schwarz sector states in heterotic and superstring theories.

Keywords: Superstrings and Heterotic Strings, Bosonic Strings

ARXIV EPRINT: 1401.7014 


\section{Contents}

1 Introduction $\quad 1$

2 A field theory example 3

2.1 The model 3

$\begin{array}{ll}2.2 & \text { The algorithm for computing the physical mass }\end{array}$

2.3 Explicit evaluation of the physical mass 9

2.4 Masses of the unphysical / pure gauge states 11

3 Organization of off-shell amplitudes in string theory $\quad 12$

$\begin{array}{lll}3.1 & \text { Off-shell string states and a basis } & 12\end{array}$

$\begin{array}{lll}3.2 & \text { Off-shell amplitudes } & 15\end{array}$

$\begin{array}{lll}3.3 & \text { Off-shell amplitudes from 1PI amplitudes } & 17\end{array}$

$4 \begin{array}{ll}\text { Physical state propagator in string theory } & 19\end{array}$

$\begin{array}{lll}4.1 & \text { Renormalized propagator at a given mass level } & 19\end{array}$

$\begin{array}{ll}4.2 & \text { An alternate definition of } \widetilde{F}_{T} \\ & 20\end{array}$

4.3 Renormalized physical state propagator and masses 21

4.4 Renormalized masses in the unphysical / pure gauge sector 23

4.5 Dependence on choice of local coordinates 24

5 Poles of S-matrix elements of massless / BPS / special states 25

$\begin{array}{lll}6 & \text { All order results } & 27\end{array}$

$\begin{array}{lll}7 & \text { Generalizations to heterotic and super string theories } & 28\end{array}$

\section{Introduction}

We now have a well defined algorithm for computing perturbative S-matrix elements of massless gauge particles and BPS states in string theory to all orders in perturbation theory [1-17]. These states have the property that their masses are not renormalized away from the tree level values due to various underlying symmetries. However string theory also contains stable and unstable particles whose masses are not protected from quantum corrections, and a direct systematic computation of the renormalized masses and S-matrix elements of these states is plagued with difficulties [18-31]. The main difficulty arises from the fact that world-sheet conformal invariance requires us to use vertex operators of dimension $(0,0)$ for defining string amplitudes, and this condition on the dimension of the operator translates to requiring the momenta to satisfy the tree level mass-shell condition. 
Thus in the presence of a mass renormalization we run into an apparent conflict between the requirement of world-sheet conformal invariance and renormalized mass-shell condition.

In a previous paper [32] we described a systematic procedure for computing the renormalized masses and S-matrix elements of a special class of states in bosonic string theory which do not mix with unphysical states under renormalization. Our goal in this paper will be to generalize this procedure to general states in bosonic string theory. We shall also briefly discuss extensions to the Neveu-Schwarz (NS) sector states in superstring and heterotic string theories.

We shall now summarize the contents of the rest of the sections. The reason that we had to restrict our analysis to a special class of states in [32] was to avoid the mixing between physical and unphysical states which are degenerate at tree level. In section 2 we construct an example of a gauge theory where the tree level spectrum in a particular gauge has accidental degeneracy between physical and unphysical states. We then develop an algorithm for extracting the quantum corrected physical mass in this theory, with the aim of generalizing this to string theory later.

In section 3 we review some basic results for on-shell states in closed bosonic string theory, dividing them into physical, unphysical and pure gauge states and discuss their off-shell generalization. We also review the prescription for defining off-shell amplitudes in string theory which depend on the choice of local coordinates at the punctures where the vertex operators are inserted. Finally we discuss the constraints imposed on the choice of local coordinate system from the requirement that they be compatible with the plumbing fixture procedure for gluing two Riemann surfaces to form a third one. This allows us to express an off-shell amplitude as sums of products of one particle irreducible contributions and propagators.

Section 4-6 contains our main results. In section 4 we generalize the method of section 2 for systematically computing the renormalized physical masses in string theory. We also show that at one loop order the renormalized physical masses are independent of the choice of local coordinate system but the renormalized masses in the unphysical / pure gauge sector do depend on the choice of local coordinates. In section 5 we examine the locations of the poles in the scattering amplitudes of external massless / BPS / special states in the complex $-k^{2}$ plane where $k$ is given by the sum of some specific subset of external momenta. We find that the possible locations of the poles are precisely at the squares of physical and unphysical masses found using the general algorithm of section 4 . We also show that at the leading order the residues at the physical poles are non-vanishing in general but the residues at the poles associated with the unphysical / pure gauge sector states vanish. In section 6 we combine the results of section 4 , section 5 with the result of [32] that the S-matrices of massless / BPS / special states are independent of the choice of local coordinate system, to argue that to all orders in string perturbation theory the renormalized physical masses are independent of the choice of local coordinate system and that the residues at the poles associated with the unphysical / pure gauge sector states vanish. In other words the poles in the S-matrix elements of massless / BPS / special states in the $-k^{2}$ plane occur only at the renormalized physical mass ${ }^{2}$ defined in section 4 .

The proof that physical masses are independent of the choice of local coordinates 
requires us to assume that the corresponding physical states appear in the intermediate channel of the S-matrix of some set of massless / BPS / special states. In the examples we have examined this always seems to hold.

Finally in section 7 we briefly discuss generalization of our analysis to Neveu-Schwarz sector states in heterotic and superstring theories.

\section{$2 \quad$ A field theory example}

In this section we shall illustrate the problem of mixing between physical and unphysical states in a gauge theory. We shall also provide an algorithm for extracting the renormalized physical mass in this theory. This algorithm will be generalized to string theory in section 4 .

\section{$2.1 \quad$ The model}

Consider a quantum field theory in $D+1$ dimensions containing an abelian gauge field $A_{\mu}$ and a pair of complex scalars $\phi, \chi$, each carrying charge $q$ under the gauge field. We consider a gauge invariant Lagrangian density of the form

$$
\begin{aligned}
\mathcal{L}= & -\frac{1}{4} F_{\mu \nu} F^{\mu \nu}-\left(\partial_{\mu} \phi^{*}+i q A_{\mu} \phi^{*}\right)\left(\partial^{\mu} \phi-i q A^{\mu} \phi\right)-c\left(\phi^{*} \phi-v^{2}\right)^{2} \\
& -\left(\partial_{\mu} \chi^{*}+i q A_{\mu} \chi^{*}\right)\left(\partial^{\mu} \chi-i q A^{\mu} \chi\right)-V(\phi, \chi), \\
F_{\mu \nu} \equiv & \partial_{\mu} A_{\nu}-\partial_{\nu} A_{\mu},
\end{aligned}
$$

where $V(\phi, \chi)$ is a potential whose detailed properties will be discussed shortly, but for now we just mention that it plays no role in the breaking of the $\mathrm{U}(1)$ gauge symmetry. Minimizing the potential in the first line we see that $|\phi|=v$ is the minimum of the potential. We choose $\phi=v$ as the vacuum expectation value of $\phi$. We now define $\phi_{R, I}, \chi_{R, I}$ via

$$
\phi=v+\frac{1}{\sqrt{2}}\left(\phi_{R}+i \phi_{I}\right), \quad \chi=\frac{1}{\sqrt{2}}\left(\chi_{R}+i \chi_{I}\right),
$$

and

$$
m \equiv \sqrt{2} q v .
$$

We now describe the choice of the potential $V(\phi, \chi)$. We require it to have the property that when expanded around the point $(\phi=v, \chi=0)$, it has an expansion of the form

$$
-\frac{1}{2} m_{0}^{2} \chi_{R}^{2}-\frac{1}{2} m^{2} \chi_{I}^{2}+\text { cubic and higher order terms in } \phi_{R}, \phi_{I}, \chi_{R}, \chi_{I},
$$

where $m_{0}$ is an arbitrary mass parameter but $m$ has been chosen to be the same quantity defined in (2.3). Using this we get, after throwing away total derivative terms,

$$
\begin{aligned}
\mathcal{L}= & -\frac{1}{2} \partial_{\mu} A_{\nu} \partial^{\mu} A^{\nu}-\frac{1}{2} m^{2} A_{\mu} A^{\mu}+\frac{1}{2}\left(\partial_{\mu} A_{\mu}-m \phi_{I}\right)^{2}-\frac{1}{2} \partial_{\mu} \phi_{I} \partial^{\mu} \phi^{I}-\frac{1}{2} m^{2} \phi_{I}^{2}-\frac{1}{2} \partial_{\mu} \phi_{R} \partial^{\mu} \phi_{R} \\
& -2 c v^{2} \phi_{R}^{2}-\frac{1}{2} \partial_{\mu} \chi_{I} \partial^{\mu} \chi^{I}-\frac{1}{2} m^{2} \chi_{I}^{2}-\frac{1}{2} \partial_{\mu} \chi_{R} \partial^{\mu} \chi^{R}-\frac{1}{2} m_{0}^{2} \chi_{R}^{2}+\text { interaction terms } .
\end{aligned}
$$


To this we add a gauge fixing term

$$
\mathcal{L}_{g f}=-\frac{1}{2}\left(\partial^{\mu} A_{\mu}-m \phi_{I}\right)^{2}
$$

so that the third term in $\mathcal{L}$ is cancelled by $\mathcal{L}_{g f}$ in the total Lagrangian density $\mathcal{L}+\mathcal{L}_{g f}$. The resulting Lagrangian has the fields $A_{\mu}, \phi_{I}$ and $\chi_{I}$ all carrying mass $m$, whereas $\phi_{R}$ and $\chi_{R}$ carry different masses.

Now if we work in the momentum space and are at the rest frame $k=\left(k^{0}, \overrightarrow{0}\right)$ then the fields $A_{i}$ transform in the vector representation of the little group SO(D) whereas the fields $A_{0}, \phi_{I}$ and $\chi_{I}$ transform in the scalar representation of the same group. At tree level the fields $A_{i}$ and $\chi_{I}$ are physical whereas the fields $A_{0}$ and $\phi_{I}$ are unphysical. ${ }^{1}$ In particular by choosing unitary gauge we can remove $A_{0}$ and $\phi_{I}$ from the spectrum. Alternatively by choosing another gauge fixing term e.g. $-\left(\partial^{\mu} A_{\mu}-m \xi \phi_{I}\right)^{2} /(2 \xi)$ with $\xi \neq 1$ we could make the unphysical fields $A_{0}$ and $\phi_{I}$ have mass different from $m$ and hence non-degenerate with the physical fields. We shall however work with $\xi=1$ and address the problems associated with the degeneracy directly since this is what we shall need to do in string theory. Our main goal will be to disentangle the physical and unphysical states after inclusion of loop corrections.

Now it is clear that under loop corrections the $\mathrm{SO}(\mathrm{D})$ vector fields $A_{i}$ cannot mix with the unphysical fields and hence they remain physical states. These are the analogs of the special states considered in [32]. However the state $\chi_{I}$ can now mix with $A_{0}$ and $\phi_{I}$. To see what kind of mixing is possible, we note that according the general principle of gauge theory the corrections must take the form of a gauge invariant term written in terms of the original variables $\phi, \chi, A_{\mu}$ together with a possible renormalizaton of the gauge fixing term. Let us suppose that quantum corrections generate a gauge invariant mass term for $\chi$ of the form $-\alpha \chi^{*} \chi$ and changes the gauge fixing term (2.6) to $-\left(\partial^{\mu} A_{\mu}-m \phi_{I}+\beta \phi_{I}+\gamma \chi_{I}\right)^{2} / 2 .^{2}$ Here, $\alpha, \beta$ and $\gamma$ are in principle computable constants which arise from loop corrections. Adding these to (2.1) we can express the quadratic terms involving $A_{\mu}, \phi_{I}$ and $\chi_{I}$ as

$$
\begin{aligned}
& -\frac{1}{2} \partial_{\mu} A_{\nu} \partial^{\mu} A^{\nu}-\frac{1}{2} m^{2} A_{\mu} A^{\mu}-\frac{1}{2} \partial_{\mu} \phi_{I} \partial^{\mu} \phi^{I}-\frac{1}{2} m^{2} \phi_{I}^{2}-\frac{1}{2} \partial_{\mu} \chi_{I} \partial^{\mu} \chi^{I}-\frac{1}{2} m^{2} \chi_{I}^{2} \\
& -\frac{1}{2} \alpha \chi_{I}^{2}-\beta \phi_{I} \partial_{\mu} A^{\mu}+\frac{1}{2}\left(2 m \beta-\beta^{2}\right) \phi_{I}^{2}-\gamma \chi_{I} \partial_{\mu} A^{\mu}-\frac{1}{2} \gamma^{2} \chi_{I}^{2}+(m-\beta) \gamma \phi_{I} \chi_{I} .
\end{aligned}
$$

In momentum space, up to overall multiplication and momentum conserving delta functions, the quadratic Lagrangian density in the $\vec{k}=0$ sector can be written as

$$
\frac{1}{2} A_{i}(-k)\left\{\left(k^{0}\right)^{2}-m^{2}\right\} A_{i}(k)+\frac{1}{2}\left(A_{0}(-k) \phi_{I}(-k) \chi_{I}(-k)\right) M\left(\begin{array}{c}
A_{0}(k) \\
\phi_{I}(k) \\
\chi_{I}(k)
\end{array}\right)
$$

\footnotetext{
${ }^{1}$ In the language that we shall develop shortly, one linear combination of these fields will be called unphysical and the other will be called pure gauge.

${ }^{2}$ We could have also changed the coefficient of the $\partial_{\mu} A^{\mu}$ inside the gauge fixing term and added other gauge invariant terms, but the corrections we have taken are sufficiently general to illustrate the basic points.
} 
where

$$
M=\left(\begin{array}{ccc}
-\left(E^{2}-m^{2}\right) & \mathbf{i} E \beta & \mathbf{i} E \gamma \\
-\mathbf{i} E \beta & E^{2}-(m-\beta)^{2} & (m-\beta) \gamma \\
-\mathbf{i} E \gamma & (m-\beta) \gamma & E^{2}-m^{2}-\gamma^{2}-\alpha
\end{array}\right), \quad E \equiv k^{0}
$$

As expected $A_{i}(k)$ 's, being special states, do not mix with other fields. In this example its mass is not affected by the quantum corrections, but this is just a consequence of the limited number of terms we have added, e.g. this could change if we had added a gauge invariant term proportional to $F_{\mu \nu} F^{\mu \nu}$ in the quantum corrections to the Lagrangian density.

Let us define the matrices

$$
\mathcal{I}=\left(\begin{array}{ccc}
-1 & 0 & 0 \\
0 & 1 & 0 \\
0 & 0 & 1
\end{array}\right), \quad \widetilde{F}_{T}=\left(\begin{array}{ccc}
0 & \mathbf{i} E \beta & \mathbf{i} E \gamma \\
-\mathbf{i} E \beta & 2 m \beta-\beta^{2} & (m-\beta) \gamma \\
-\mathbf{i} E \gamma & (m-\beta) \gamma & -\gamma^{2}-\alpha
\end{array}\right)
$$

so that we can write

$$
M=-\left\{\left(m^{2}-E^{2}\right) \mathcal{I}-\widetilde{F}_{T}\right\} .
$$

The full propagator (up to overall sign and factors of $i$ ) is then given by

$$
\mathcal{V}_{T}=-M^{-1}=\left\{\left(m^{2}-E^{2}\right) \mathcal{I}-\widetilde{F}_{T}\right\}^{-1},
$$

and the renormalized squared masses are the locations of the poles of this matrix in the $E^{2}$ plane. Only one of these poles is physical. We need to find a systematic algorithm for determining which one is physical and calculate its location. This will be done in section 2.2 , but to facilitate the analysis we shall now introduce a few notations.

Let us introduce a set of basis states as follows:

$$
|p\rangle=\left(\begin{array}{l}
0 \\
0 \\
1
\end{array}\right), \quad|g\rangle=\frac{1}{|E| \sqrt{2}}\left(\begin{array}{c}
-\mathbf{i} E \\
|E| \\
0
\end{array}\right), \quad|u\rangle=\frac{1}{|E| \sqrt{2}}\left(\begin{array}{c}
\mathbf{i} E \\
|E| \\
0
\end{array}\right) .
$$

The conjugate basis $\langle p|,\langle g|$ and $\langle u|$ are defined by taking transpose together with a change of sign of the momentum vector. The latter operation changes the sign of $E$ and hence effectively the conjugate basis corresponds to hermitian conjugates of the vectors (2.13). Then we have the following identities

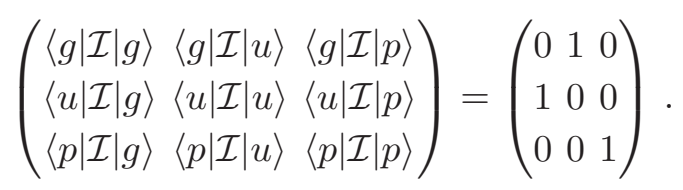

We shall call $|p\rangle,|g\rangle$ and $|u\rangle$ as tree level physical, pure gauge and unphysical states respectively. The name pure gauge for $|g\rangle$ stems from the fact that on-shell (at $|E|=m$ ) this describes a pure gauge deformation of the vacuum at the linearized level and the name physical originates from the fact that the $\chi_{I}$ field represented by the vector $|p\rangle$ is the physical field at the tree level. 


\subsection{The algorithm for computing the physical mass}

Our goal will be to develop an algorithm for finding the corrected physical state and the physical mass after taking into account the quantum correction to $M$ represented by $\widetilde{F}_{T}$. Furthermore instead of aiming at the exact result we want to do this perturbatively in the parameters $\alpha, \beta, \gamma$ since this is what we need in string theory. The problem is made complicated by the fact that the full matrix $M$ is expected to have zero eigenvalue at more than one value of $E$ near $m$, and we expect only one of these to represent physical mass. Let $m_{p}$ be the quantum corrected physical mass, and $|p\rangle^{\prime}$ be the eigenvector with zero eigenvalue at $E=m_{p}$. Then naively we might expect that as we switch off the perturbation parameters $\alpha, \beta, \gamma$, the vector $|p\rangle^{\prime}$ should approach the unperturbed physical state $|p\rangle$ and we can use this as a criterion for identifying the quantum corrected physical state. The problem however is that since the unperturbed matrix has three different eigenvectors with zero eigenvalue at $E=m$, what we have here is an analog of degenerate perturbation theory and there is no guarantee that the eigenvectors of the quantum corrected matrix will approach a particular unperturbed eigenvector in the limit of switching off the perturbation. Indeed, we shall see that in general it is not possible to construct an eigenvector with zero eigenvalue in the perturbed theory that approaches the particular vector $|p\rangle$ in the limit $\alpha, \beta, \gamma \rightarrow 0$. The best we can do is to find such an eigenvector that approaches a linear combination of the unperturbed physical state $|p\rangle$ and the unperturbed pure gauge state $|g\rangle$ as we switch off the perturbation. We shall take this as the criterion for identifying the quantum corrected physical state and look for an algorithm for constructing such a state.

With this goal in mind, we now seek a change of basis of the form

$$
|p\rangle^{\prime}=A|p\rangle+B|g\rangle+C|u\rangle, \quad|g\rangle^{\prime}=|g\rangle+D|p\rangle, \quad|u\rangle^{\prime}=|u\rangle+K|p\rangle,
$$

such that the following conditions hold

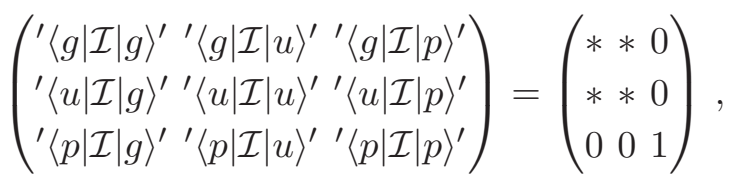

and

$$
\left(\begin{array}{l}
\left.{ }^{\prime}\left\langle g\left|\widetilde{F}_{T}\right| g\right\rangle^{\prime}{ }^{\prime}\left\langle g\left|\widetilde{F}_{T}\right| u\right\rangle^{\prime}{ }^{\prime}\left\langle g\left|\widetilde{F}_{T}\right| p\right\rangle\right\rangle^{\prime} \\
{ }^{\prime}\left\langle u\left|\widetilde{F}_{T}\right| g\right\rangle^{\prime}{ }^{\prime}\left\langle u\left|\widetilde{F}_{T}\right| u\right\rangle^{\prime}{ }^{\prime}\left\langle u\left|\widetilde{F}_{T}\right| p\right\rangle^{\prime} \\
{ }^{\prime}\left\langle p\left|\widetilde{F}_{T}\right| g\right\rangle^{\prime}{ }^{\prime}\left\langle p\left|\widetilde{F}_{T}\right| u\right\rangle^{\prime}{ }^{\prime}\left\langle p\left|\widetilde{F}_{T}\right| p\right\rangle^{\prime}
\end{array}\right)=\left(\begin{array}{ccc}
* * & 0 \\
* & * 0 & 0 \\
0 & 0 & *
\end{array}\right) .
$$

where $*$ denotes unconstrained numbers. Notice that $(2.15)$ is not the most general change of basis. In fact the most general change of basis is related to the one given in (2.15) by arbitrary mixing between the states $|u\rangle^{\prime}$ and $|g\rangle^{\prime}$ without involving $|p\rangle^{\prime}$. However all the conditions demanded in (2.16), (2.17) are invariant under such a change of basis and hence by taking convenient linear combinations of $|u\rangle^{\prime}$ and $|g\rangle^{\prime}$ satisfying (2.16), (2.17) we can always ensure that the change of basis is of the form given in (2.15). We now 
substitute (2.15) into (2.16), (2.17) and use (2.14) to get

$$
\begin{array}{r}
A^{*} A+B^{*} C+C^{*} B=1, \quad D^{*} A+C=0, \quad K^{*} A+B=0, \\
A\left\langle u\left|\widetilde{F}_{T}\right| p\right\rangle+B\left\langle u\left|\widetilde{F}_{T}\right| g\right\rangle+C\left\langle u\left|\widetilde{F}_{T}\right| u\right\rangle+K^{*} A\left\langle p\left|\widetilde{F}_{T}\right| p\right\rangle+K^{*} B\left\langle p\left|\widetilde{F}_{T}\right| g\right\rangle+K^{*} C\left\langle p\left|\widetilde{F}_{T}\right| u\right\rangle=0 \\
A\left\langle g\left|\widetilde{F}_{T}\right| p\right\rangle+B\left\langle g\left|\widetilde{F}_{T}\right| g\right\rangle+C\left\langle g\left|\widetilde{F}_{T}\right| u\right\rangle+D^{*} A\left\langle p\left|\widetilde{F}_{T}\right| p\right\rangle+D^{*} B\left\langle p\left|\widetilde{F}_{T}\right| g\right\rangle+D^{*} C\left\langle p\left|\widetilde{F}_{T}\right| u\right\rangle=0 .
\end{array}
$$

We shall soon discuss how to construct $A, B, C, D, K$ perturbatively satisfying (2.18) and the criteria mentioned at the beginning of this subsection. However let us first examine the consequences of (2.16) and (2.17). Using these equations we see that in the primed basis the matrices $\mathcal{I}$ and $\widetilde{F}_{T}$ are exactly block diagonal, with the $|p\rangle^{\prime}$ block having no mixing with the $|u\rangle^{\prime}$ and $|g\rangle^{\prime}$ blocks. Of course the basis we have chosen is not orthonormal in the $\left(|u\rangle^{\prime},|g\rangle^{\prime}\right)$ sector, but this can be rectified by appropriate linear transformation in the $\left(|u\rangle^{\prime},|g\rangle^{\prime}\right)$ space without affecting the $|p\rangle^{\prime}-|p\rangle^{\prime}$ element. Thus we get

$$
{ }^{\prime}\left\langle p\left|\mathcal{V}_{T}\right| p\right\rangle^{\prime}=\left\{\left(m^{2}-E^{2}\right)-\widetilde{F}(E)\right\}^{-1},
$$

where

$$
\begin{aligned}
\widetilde{F}(E) \equiv & '\left\langle p\left|\widetilde{F}_{T}\right| p\right\rangle^{\prime} \\
= & A^{*} A\left\langle p\left|\widetilde{F}_{T}\right| p\right\rangle+A^{*} B\left\langle p\left|\widetilde{F}_{T}\right| g\right\rangle+A^{*} C\left\langle p\left|\widetilde{F}_{T}\right| u\right\rangle+B^{*} A\left\langle g\left|\widetilde{F}_{T}\right| p\right\rangle+B^{*} B\left\langle g\left|\widetilde{F}_{T}\right| g\right\rangle \\
& +B^{*} C\left\langle g\left|\widetilde{F}_{T}\right| u\right\rangle+C^{*} A\left\langle u\left|\widetilde{F}_{T}\right| p\right\rangle+C^{*} B\left\langle u\left|\widetilde{F}_{T}\right| g\right\rangle+C^{*} C\left\langle u\left|\widetilde{F}_{T}\right| u\right\rangle .
\end{aligned}
$$

The pole of (2.19) can be constructed iteratively by expressing this equation as

$$
E^{2}=m^{2}-\widetilde{F}(E),
$$

and solving the equation iteratively by starting with $E^{2}=m^{2}$. We can identify this as the physical pole provided the following two conditions hold:

1. Let us introduce a perturbation parameter $\lambda$ and take

$$
\alpha \sim \lambda, \quad \beta \sim \lambda, \quad \gamma \sim \lambda
$$

In particular if $\alpha, \beta, \gamma$ arise at one loop order then the power of $\lambda$ counts the number of loops. We need to ensure that the coefficient of $\lambda^{n}$ in the expressions for $A, \cdots K$ and $\widetilde{F}(E)$ are free from any pole at $E \simeq m$ for every $n$. Otherwise the iterative procedure for finding the solution that starts with $E=m$ will break down.

2. We also need to ensure that the coefficient $C$ approaches 0 in the limit $\lambda \rightarrow 0$ and $E \rightarrow m$ so that the state $|p\rangle^{\prime}$ approaches a linear combination of the tree level physical state and tree level pure gauge state in this limit. $|p\rangle^{\prime}$ will then satisfy the criteria mentioned at the beginning of this subsection.

We shall now discuss how to solve (2.18) satisfying these conditions. Since each matrix element of $\widetilde{F}_{T}$ is of order $\lambda$, we can factor out the overall factor of $\lambda$ from the last two 
equations in (2.18), take the $\lambda \rightarrow 0$ limit, and regard (2.18) as a set of $\lambda$ independent equations which can be solved to determine the leading order result for the coefficients $A, \cdots K$. It is easy to check that leaving aside an overall phase there are as many unknowns as the number of equations, and hence we expect these equations to have solutions. Solving the leading order equations can in fact be facilitated by using another expansion parameter, namely $\left(E^{2}-m^{2}\right)$. For this we note that $(2.10),(2.13)$ gives

$$
\begin{aligned}
& \lambda^{-1}\left\langle p\left|\widetilde{F}_{T}\right| g\right\rangle \sim \mathcal{O}\left(E^{2}-m^{2}\right)+\mathcal{O}(\lambda), \quad \lambda^{-1}\left\langle g\left|\widetilde{F}_{T}\right| p\right\rangle \sim \mathcal{O}\left(E^{2}-m^{2}\right)+\mathcal{O}(\lambda), \\
& \lambda^{-1}\left\langle g\left|\widetilde{F}_{T}\right| g\right\rangle \sim \mathcal{O}\left(E^{2}-m^{2}\right)+\mathcal{O}(\lambda)
\end{aligned}
$$

while the other matrix elements of $\lambda^{-1} \widetilde{F}_{T}$ are of order unity as $E \rightarrow m$ and $\lambda \rightarrow 0$. Making use of (2.23), let us look for a leading order in $\lambda$ solution in which

$$
A, B, K \sim 1, \quad C, D \sim\left(E^{2}-m^{2}\right) .
$$

Using (2.23), (2.24) we see that to the leading order in $\lambda,(2.18)$ gives

$$
\begin{aligned}
& A^{*} A=1+\mathcal{O}\left(E^{2}-m^{2}\right), \quad D^{*} A+C=0, \quad K^{*} A+B=0, \\
& \lambda^{-1}\left\{A\left\langle u\left|\widetilde{F}_{T}\right| p\right\rangle+B\left\langle u\left|\widetilde{F}_{T}\right| g\right\rangle+K^{*} A\left\langle p\left|\widetilde{F}_{T}\right| p\right\rangle\right\}=\mathcal{O}\left(E^{2}-m^{2}\right), \\
& \lambda^{-1}\left\{A\left\langle g\left|\widetilde{F}_{T}\right| p\right\rangle+B\left\langle g\left|\widetilde{F}_{T}\right| g\right\rangle+C\left\langle g\left|\widetilde{F}_{T}\right| u\right\rangle+D^{*} A\left\langle p\left|\widetilde{F}_{T}\right| p\right\rangle\right\}=\mathcal{O}\left(\left(E^{2}-m^{2}\right)^{2}\right) .
\end{aligned}
$$

Each term in the left hand side of the first, third and fourth equations is of order unity and each term in the left hand side of the third and fifth equations is of order $\left(E^{2}-m^{2}\right)$. The solution is

$$
\begin{aligned}
& A=1+\mathcal{O}\left(E^{2}-m^{2}\right), \quad K^{*}=\left\{\left\langle u\left|\widetilde{F}_{T}\right| g\right\rangle-\left\langle p\left|\widetilde{F}_{T}\right| p\right\rangle\right\}^{-1}\left\langle u\left|\widetilde{F}_{T}\right| p\right\rangle+\mathcal{O}\left(E^{2}-m^{2}\right), \\
& D^{*}=\left\{\left\langle g\left|\widetilde{F}_{T}\right| u\right\rangle-\left\langle p\left|\widetilde{F}_{T}\right| p\right\rangle\right\}^{-1}\left\{\left\langle g\left|\widetilde{F}_{T}\right| p\right\rangle-K^{*}\left\langle g\left|\widetilde{F}_{T}\right| g\right\rangle\right\}+\mathcal{O}\left(\left(E^{2}-m^{2}\right)^{2}\right), \\
& B=-K^{*}+\mathcal{O}\left(E^{2}-m^{2}\right), \quad C=-D^{*}+\mathcal{O}\left(\left(E^{2}-m^{2}\right)^{2}\right) .
\end{aligned}
$$

Using (2.23) and the comments below it, we see that as long as the order $\lambda$ contribution to $\left\{\left\langle u\left|\widetilde{F}_{T}\right| g\right\rangle-\left\langle p\left|\widetilde{F}_{T}\right| p\right\rangle\right\}$ does not vanish (and in particular does not have zero at $E^{2}=m^{2}$ ), $A, B$ and $K$ given in (2.26) are of order unity, while $C$ and $D$ are of order $\left(E^{2}-m^{2}\right)$, in agreement with our assumption (2.24). The reader may be surprised by the appearance of the one loop term $\left\{\left\langle u\left|\widetilde{F}_{T}\right| g\right\rangle-\left\langle p\left|\widetilde{F}_{T}\right| p\right\rangle\right\}$ in the denominator in a perturbation theory, but this is simply a consequence of the degenerate perturbation theory that we need to carry out in this case. Requiring $\left\{\left\langle u\left|\widetilde{F}_{T}\right| g\right\rangle-\left\langle p\left|\widetilde{F}_{T}\right| p\right\rangle\right\}$ to be non-zero is equivalent to demanding that the degeneracy between the physical and the unphysical / pure gauge states is lifted at the first order. Starting with (2.26) we can now iteratively solve the system of equations in a power series in $\lambda$ and $\left(E^{2}-m^{2}\right)$. For this we choose $A$ to be real, ${ }^{3}$ express eqs. (2.18)

\footnotetext{
${ }^{3}$ Eqs. (2.18) have a symmetry under which the constants $A, B, C$ are multiplied by an overall phase. We have chosen this phase appropriately to make $A$ real.
} 


$$
\begin{aligned}
A= & \sqrt{1-B^{*} C-C^{*} B} \\
K^{*}= & \left\{\left\langle u\left|\widetilde{F}_{T}\right| g\right\rangle-\left\langle p\left|\widetilde{F}_{T}\right| p\right\rangle\right\}^{-1}\left[A\left\langle u\left|\widetilde{F}_{T}\right| p\right\rangle+\left(B+K^{*}\right)\left\langle u\left|\widetilde{F}_{T}\right| g\right\rangle+C\left\langle u\left|\widetilde{F}_{T}\right| u\right\rangle\right. \\
& \left.+K^{*}(A-1)\left\langle p\left|\widetilde{F}_{T}\right| p\right\rangle+K^{*} B\left\langle p\left|\widetilde{F}_{T}\right| g\right\rangle+K^{*} C\left\langle p\left|\widetilde{F}_{T}\right| u\right\rangle\right], \\
D^{*}= & \left\{\left\langle g\left|\widetilde{F}_{T}\right| u\right\rangle-\left\langle p\left|\widetilde{F}_{T}\right| p\right\rangle\right\}^{-1}\left[A\left\langle g\left|\widetilde{F}_{T}\right| p\right\rangle+B\left\langle g\left|\widetilde{F}_{T}\right| g\right\rangle+\left(C+D^{*}\right)\left\langle g\left|\widetilde{F}_{T}\right| u\right\rangle\right. \\
& \left.+D^{*}(A-1)\left\langle p\left|\widetilde{F}_{T}\right| p\right\rangle+D^{*} B\left\langle p\left|\widetilde{F}_{T}\right| g\right\rangle+D^{*} C\left\langle p\left|\widetilde{F}_{T}\right| u\right\rangle\right], \\
C= & -D^{*} A, \quad B=-K^{*} A,
\end{aligned}
$$

and evaluate the right hand sides of these equations iteratively, beginning with the leading order solution. To get a perturbation expansion we also need to expand $\left\{\left\langle u\left|\widetilde{F}_{T}\right| g\right\rangle-\right.$ $\left.\left\langle p\left|\widetilde{F}_{T}\right| p\right\rangle\right\}^{-1}$ in a power series in $\lambda$ starting with the leading order solution. Each power of $\lambda$ will be free from any pole near $E^{2}=m^{2}$ as long as the leading order result for $\lambda^{-1}\left\{\left\langle u\left|\widetilde{F}_{T}\right| g\right\rangle-\left\langle p\left|\widetilde{F}_{T}\right| p\right\rangle\right\}$ does not have any zero near $E^{2}=m^{2}$. Once we determine the coefficients $A, \cdots K$ we can also determine $\widetilde{F}(E)$ using $(2.20)$.

Note that in this scheme even in a fixed order in $\lambda$ we need to iterate the procedure infinite number of times to generate all powers of $E^{2}-m^{2}$. However eventually we are interested in computing these coefficients at the physical mass ${ }^{2}$ which differs from $m^{2}$ by order $\lambda$. Similarly when we solve $(2.21)$ to find the location of the pole, we need to know the expansion of $\widetilde{F}(E)$ to order $\left(E^{2}-m^{2}\right)^{n}$ for computing the correction to mass ${ }^{2}$ to order $\lambda^{n+1}$. Thus for computing physical quantities to any given order in $\lambda$ we need to run the iteration only a finite number of times.

We now observe that since eq. (2.26) gives $B \simeq-K^{*} \sim 1$, it follows from (2.15) that $|p\rangle^{\prime}$ differs from $|p\rangle$ by an order one term proportional to the pure gauge states. This is a consequence of having degenerate eigenvalues at the tree level and will continue to be true in string theory as well. On the other hand since $C \sim E^{2}-m^{2}$ which is of order $\lambda$ when $E$ is set equal to the corrected physical mass, the coefficient of $|u\rangle$ in $|p\rangle^{\prime}$ vanishes as $\lambda \rightarrow 0$. Thus the quantum corrected physical state approaches a linear combination of the unperturbed physical state and the unperturbed pure gauge state in the limit in which we switch off the perturbation. This is consistent with the criteria for identifying the quantum corrected physical state that we set out at the beginning of this subsection.

\subsection{Explicit evaluation of the physical mass}

Let us now explicitly evaluate the coefficients $A, \cdots K$ and $F(E)$ for the problem at hand and from this find the location of the physical pole. From (2.10), (2.13) it follows that here

$$
\begin{aligned}
\left\langle p\left|\widetilde{F}_{T}\right| p\right\rangle & =-\gamma^{2}-\alpha, \\
\left\langle g\left|\widetilde{F}_{T}\right| g\right\rangle & =\frac{\beta}{2}(2 m-\beta-2|E|), \\
\left\langle u\left|\widetilde{F}_{T}\right| u\right\rangle & =\frac{\beta}{2}(2 m-\beta+2|E|), \\
\left\langle p\left|\widetilde{F}_{T}\right| g\right\rangle & =\left\langle g\left|\widetilde{F}_{T}\right| p\right\rangle=\frac{1}{\sqrt{2}}(-|E|+m-\beta) \gamma,
\end{aligned}
$$




$$
\begin{aligned}
& \left\langle p\left|\widetilde{F}_{T}\right| u\right\rangle=\left\langle u\left|\widetilde{F}_{T}\right| p\right\rangle=\frac{1}{\sqrt{2}}(|E|+m-\beta) \gamma, \\
& \left\langle g\left|\widetilde{F}_{T}\right| u\right\rangle=\left\langle u\left|\widetilde{F}_{T}\right| g\right\rangle=\frac{\beta}{2}(2 m-\beta),
\end{aligned}
$$

This gives the leading order solutions (2.26) to be

$$
\begin{aligned}
& A=1, \quad B=-K=-\frac{1}{\sqrt{2}}(\beta m+\alpha)^{-1} \gamma(m+|E|) \\
& C=-D=-(\beta m+\alpha)^{-1}\left\{\frac{1}{\sqrt{2}}(-|E|+m) \gamma-\frac{\beta \gamma}{\sqrt{2}}\left(m^{2}-E^{2}\right)(\beta m+\alpha)^{-1}\right\} .
\end{aligned}
$$

There are corrections to these solutions of order $\lambda$ and also of order $\left(E^{2}-m^{2}\right)\left(\left(E^{2}-m^{2}\right)^{2}\right.$ in $C$ and $D$ ), but these will not be needed for computing the leading correction to the physical mass. Since $\alpha, \beta, \gamma$ are each of order $\lambda$ we see that $B \simeq-K^{*} \sim 1$ and $C \simeq-D^{*} \sim(|E|-m)$ in the $\lambda \rightarrow 0$ limit, in agreement with the general results quoted earlier. Substituting these into (2.20) and using (2.28) we get

$$
\widetilde{F}(E)=-\alpha+\mathcal{O}\left(\lambda^{2}\right)+\mathcal{O}(\lambda)(|E|-m) .
$$

The iterative procedure (2.21) now gives the leading order correction to the physical mass

$$
E^{2}=m^{2}+\alpha+\mathcal{O}\left(\lambda^{2}\right) .
$$

The physical state at leading order in $\lambda$, obtained from (2.13), (2.15), (2.25) and (2.29) is given by, for $E=\sqrt{m^{2}+\alpha}$

$$
\left(\begin{array}{c}
i \gamma E /(\alpha+\beta m) \\
-\gamma m /(\alpha+\beta m) \\
1
\end{array}\right)+\mathcal{O}(\lambda)
$$

Let us compare this with the exact result. We have from (2.9)

$$
\operatorname{det} M=-\left(E^{2}-m^{2}+m \beta\right)^{2}\left(E^{2}-m^{2}-\alpha\right) .
$$

This has zeroes at $E^{2}=m^{2}+\alpha$ and $E^{2}=m^{2}-m \beta$. Since we know that $\beta$ enters through the renormalized gauge fixing term, the physical mass should not depend on $\beta$. This determines the physical pole to be at

$$
E^{2}=m^{2}+\alpha
$$

which agrees with the perturbative result (2.31). Furthermore at $E=\sqrt{m^{2}+\alpha}$ we can easily compute the zero eigenvector of $M$ and it is given by

$$
v=\left(\begin{array}{c}
i \gamma E /(\alpha+\beta m) \\
-\gamma m /(\alpha+\beta m) \\
1
\end{array}\right) .
$$

This agrees with the perturbative result (2.32) up to corrections of order $\lambda$. 


\subsection{Masses of the unphysical / pure gauge states}

For completeness we shall also describe the computation of the masses in the unphysical / pure gauge sector using perturbation theory. For this we define the matrices

$$
\mathcal{I}^{\prime}=\left(\begin{array}{cc}
{ }^{\prime}\langle g|\mathcal{I}| g\rangle^{\prime} & { }^{\prime}\langle g|\mathcal{I}| u\rangle^{\prime} \\
\prime\langle u|\mathcal{I}| g\rangle^{\prime} & { }^{\prime}\langle u|\mathcal{I}| u\rangle^{\prime}
\end{array}\right), \quad \widetilde{F}^{\prime}=\left(\begin{array}{cc}
{ }^{\prime}\left\langle g\left|\widetilde{F}_{T}\right| g\right\rangle^{\prime} & { }^{\prime}\left\langle g\left|\widetilde{F}_{T}\right| u\right\rangle^{\prime} \\
{ }^{\prime}\left\langle u\left|\widetilde{F}_{T}\right| g\right\rangle^{\prime} & { }^{\prime}\left\langle u\left|\widetilde{F}_{T}\right| u\right\rangle^{\prime}
\end{array}\right) .
$$

Then the unphysical / pure gauge sector masses will be at the zeroes of the eigenvalues of the matrix

$$
\left(m^{2}-E^{2}\right) \mathcal{I}^{\prime}-\widetilde{F}^{\prime}(E),
$$

as a function of $E .^{4}$ For computing the first subleading correction to the unphysical mass we can use the ansatz that the zero eigenvalue of (2.37) will occur at $(E-m) \sim \lambda$ and evaluate each matrix element to order $\lambda$ using this ansatz. Since $\left(m^{2}-E^{2}\right) \sim \lambda$ we have to evaluate $\mathcal{I}^{\prime}$ to order unity. It follows from (2.15) and the fact that at the leading order $D \sim\left(E^{2}-m^{2}\right) \sim \lambda$ that $\mathcal{I}^{\prime}$ to order $\lambda^{0}$ has the structure

$$
\left(\begin{array}{cc}
0 & 1 \\
1 & K^{*} K
\end{array}\right)
$$

with $K$ given in (2.26). On the other hand (2.15), (2.23) and (2.24) shows that $\widetilde{F}^{\prime}$ to order $\lambda$ has the structure

$$
\left(\begin{array}{cc}
0 & \left\langle g\left|\widetilde{F}_{T}\right| u\right\rangle \\
\left\langle u\left|\widetilde{F}_{T}\right| g\right\rangle & '\left\langle u\left|\widetilde{F}_{T}\right| u\right\rangle^{\prime}
\end{array}\right)
$$

Thus for computing order $\lambda$ correction to the unphysical / pure gauge sector masses we need to look for zero eigenvalue of the matrix

$$
\left(\begin{array}{cc}
0 & \left(m^{2}-E^{2}\right)-\left\langle g\left|\widetilde{F}_{T}\right| u\right\rangle \\
\left(m^{2}-E^{2}\right)-\left\langle u\left|\widetilde{F}_{T}\right| g\right\rangle & \left(m^{2}-E^{2}\right) K^{*} K-{ }^{\prime}\left\langle u\left|\widetilde{F}_{T}\right| u\right\rangle^{\prime}
\end{array}\right) .
$$

Now in order that a matrix has zero eigenvalue, its determinant must vanish. From the structure of the matrix given above it is clear that this requires one of the off-diagonal elements to vanish. Since the off-diagonal elements are conjugates of each other and hence vanish at the same value of $E$, the condition for zero eigenvalue of the (2.40) can be stated as

$$
\left(m^{2}-E^{2}\right)-\left\langle u\left|\widetilde{F}_{T}\right| g\right\rangle=0 .
$$

Using the value of $\left\langle u\left|\widetilde{F}_{T}\right| g\right\rangle$ quoted in (2.28) we see that to order $\lambda$ the renormalized masses in the unphysical / pure gauge sector occur at the zero of

$$
E^{2}-m^{2}+m \beta=0 .
$$

This is in agreement with the exact result quoted below (2.33).

\footnotetext{
${ }^{4}$ It follows from $(2.15),(2.24)$ that $\mathcal{I}^{\prime}$ is a non-singular matrix near $E \sim m$ and hence the zero eigenvalue of (2.37) occurs at the same value of $E$ as that of $m^{2}-E^{2}-\left(\mathcal{I}^{\prime}\right)^{-1} \widetilde{F}^{\prime}(E)$.
} 


\section{Organization of off-shell amplitudes in string theory}

In this section we shall discuss some general aspects of off-shell states and off-shell amplitudes in closed bosonic string theory.

\subsection{Off-shell string states and a basis}

We begin by describing the space of off-shell string states with which we shall work and reviewing some well known results about the choice of basis for off-shell states. Off-shell string states are required to satisfy the following conditions:

1. They have ghost number 2 where we count the $c, \bar{c}$ ghosts to have ghost number 1 , $b, \bar{b}$ ghosts to have ghost number -1 and $\operatorname{SL}(2, \mathrm{C})$ invariant vacuum to have ghost number 0 .

2. They are annihilated by the $b, \bar{b}$ ghost zero modes $b_{0}$ and $\bar{b}_{0}$ and $L_{0}-\bar{L}_{0}$ where $\bar{L}_{n}$ and $L_{n}$ are the total left and right moving Virasoro generators.

This is also the space of off-shell states in covariant closed string field theory in the Siegel gauge [33]. The requirement of annihilation by $\left(L_{0}-\bar{L}_{0}\right)$ and $\left(b_{0}-\bar{b}_{0}\right)$ is needed for consistently defining off-shell amplitude [34] whereas the condition $\left(b_{0}+\bar{b}_{0}\right) \mid$ state $\rangle=0$ is needed to make the kinetic operator invertible. ${ }^{5}$ In this space we can introduce a nondegenerate inner product between states $|s\rangle$ and $\left|s^{\prime}\right\rangle$ via

$$
\left\langle s \mid s^{\prime}\right\rangle \equiv\left\langle s\left|c_{0} \bar{c}_{0}\right| s^{\prime}\right\rangle_{B P Z}
$$

where $\left\langle r \mid r^{\prime}\right\rangle_{B P Z}$ is the BPZ inner product. In defining the bra $\langle r|$ corresponding to a given ket $|r\rangle$ we reverse the sign of the momentum. We also remove the momentum conserving delta function from the definition of the inner product. The fact that the inner product is non-degenerate follows from the Fock space representation of the basis states.

On-shell condition for the string state $|s\rangle$ takes the form

$$
L_{0}|s\rangle=0,
$$

which also implies $\bar{L}_{0}|s\rangle=0$. On-shell we can divide the states into physical, pure gauge and unphysical states as follows. First of all pure gauge states are of the form

$$
Q_{B}|r\rangle
$$

\footnotetext{
${ }^{5}$ In contrast the off-shell states in gauge invariant closed string field theory of [33] are only annihilated by $\left(b_{0}-\bar{b}_{0}\right)$ and $\left(L_{0}-\bar{L}_{0}\right)$. Like in all gauge theories, the kinetic operator in this theory is not invertible till we fix a gauge and the Siegel gauge condition of annihilation by $\left(b_{0}+\bar{b}_{0}\right)$ precisely does that. In quantum closed string field theory we also need to relax the constraint on the ghost number and allow states of all ghost numbers to propagate in the loop. In our analysis we shall dump all the loop contributions into one particle irreducible (1PI) amplitudes and express the full amplitude as sum of tree diagrams constructed out of 1PI amplitudes as vertices and tree level propagators. Thus the only place where we have to explicitly introduce off-shell states is as the external lines of the 1PI amplitudes and as the states propagating along the propagator in the tree amplitudes. These states always carry ghost number two when we compute physical amplitudes relevant for mass renormalization or S-matrix elements, and hence we have put that restriction on the definition of off-shell states.
} 
where $Q_{B}$ is the total BRST charge (left moving plus right moving) and $|r\rangle$ is a state of ghost number 1 annihilated by $b_{0}, \bar{b}_{0}, L_{0}$ and $\bar{L}_{0}$. Since $Q_{B}$ has ghost number 1 , commutes with $L_{n}, \bar{L}_{n}$ and $\left\{Q_{B}, b_{0}\right\}=L_{0}$ and $\left\{Q_{B}, \bar{b}_{0}\right\}=\bar{L}_{0}$, it follows that $Q_{B}|r\rangle$ has ghost number 2 and is annihilated by $b_{0}, \bar{b}_{0}, L_{0}$ and $\bar{L}_{0}$.

Physical states are defined to be states of ghost number two which are annihilated by $Q_{B}, b_{0}, \bar{b}_{0}, L_{0}$ and $\bar{L}_{0}$ but cannot be written in the form $Q_{B}|r\rangle$ with $|r\rangle$ annihilated by $b_{0}$, $\bar{b}_{0}, L_{0}$ and $\bar{L}_{0}$. It follows from this that the physical states are orthogonal to pure gauge states. The main point to note is that $\left\{Q_{B}, c_{0}\right\}$ and $\left\{Q_{B}, \bar{c}_{0}\right\}$ do not have any $c_{0}$ or $\bar{c}_{0}$ factor, and hence the matrix elements of $\left\{Q_{B}, c_{0}\right\}$ and $\left\{Q_{B}, \bar{c}_{0}\right\}$ between states, satisfying condition 2 above, vanish. The same argument, together with the relation $Q_{B}^{2}=0$, shows that the pure gauge states also have vanishing inner product with pure gauge states. A linearly independent basis of physical states is the maximal set of physical states satisfying the condition that no linear combination of these basis states is a pure gauge state.

Now since the inner product is non-degenerate there must exist states which have nonvanishing inner product with the pure gauge states. These states are annihilated by $b_{0}, \bar{b}_{0}$, $L_{0}$ and $\bar{L}_{0}$, but not by $Q_{B}$. We shall call them unphysical states. We can choose a linearly independent basis of unphysical states such that no linear combination is annihilated by $Q_{B}$. The number of such basis states must be at least equal to the number of pure gauge states so that we have a non-degenerate inner product matrix. We shall now argue that the number is actually equal to the number of pure gauge states. For this let us temporarily relax the constraint on the ghost number and consider states of all ghost number annihilated by $b_{0}, \bar{b}_{0}, L_{0}$ and $\bar{L}_{0}$. Then since for every unphysical state $|s\rangle$ of ghost number $g, Q_{B}|s\rangle$ is a pure gauge state of ghost number $g+1$, we conclude that the number of pure gauge states at ghost number $g+1$ is the same as the number of unphysical states at ghost number $g$. On the other hand, since the inner product (3.1) pairs states of ghost number $g$ and $4-g$, we know from our previous argument that the number of unphysical states at ghost number $3-g$ must be at least equal to the number of pure gauge states at ghost number $g+1$ and hence the number of unphysical states at ghost number $g$. Taking $g \rightarrow 3-g$ we can arrive at the reverse conclusion. This shows that the number of unphysical states at ghost number $3-g$ should be equal to the number of unphysical states at ghost number $g$ and hence the number of pure gauge states at ghost number $g+1$. Taking $g=1$ we see that the number of unphysical states at ghost number 2 must be equal to the number of pure gauge states at ghost number 2 . This is the promised result.

Let us now return to states of ghost number 2 only. We have already seen that the inner product pairs unphysical states with pure gauge states by a non-degenerate matrix and that the pure gauge states are orthogonal to themselves as well as physical states. By adding appropriate linear combinations of pure gauge states and physical states to the unphysical states we can ensure that the latter are orthonormal to the physical states and unphysical states. Taking further linear combinations within physical states and within unphysical states we can ensure that the physical states form an orthonormal basis and that the pure gauge states and the unphysical states are paired in a one to one fashion. Thus at any mass level the inner product matrix will have a block diagonal structure of 
the form

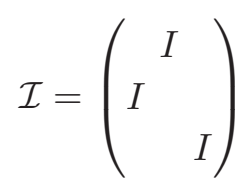

where $I$ denotes identity matrix of appropriate dimensions. The first set of rows/columns stand for pure gauge states, the second set of rows/columns stand for unphysical states and the last set of rows/columns stand for physical states. At non-zero momentum, it is in fact possible to choose a basis satisfying this requirement with physical states of the form

$$
|\alpha\rangle=c_{1} \bar{c}_{1}\left|\Phi_{\alpha}\right\rangle
$$

where $\Phi_{\alpha}$ are dimension $(1,1)$ primary in the matter sector satisfying

$$
\langle\alpha \mid \beta\rangle \equiv\left\langle\Phi_{\alpha}\left|c_{-1} \bar{c}_{-1} c_{0} \bar{c}_{0} c_{1} \bar{c}_{1}\right| \Phi_{\beta}\right\rangle_{B P Z}=\delta_{\alpha \beta} .
$$

Physical states of the form (3.5) are dimension zero primaries and hence transform as scalars under conformal transformation.

So far we have reviewed well known results, but now we shall make a small jump and discuss the off-shell continuation of these results. At a given mass level $m$ we can go off-shell (satisfying the two conditions mentioned at the beginning of this section) by deforming the momentum $k$ such that $k^{2}+m^{2}$ is deformed away from zero. We shall require the deformed basis to still satisfy the inner product structure described in (3.4), but will need to relax the various other requirements by terms of order $\left(k^{2}+m^{2}\right)$. For example if we take a state $|s\rangle$ of ghost number 1 that is annihilated by $b_{0}, \bar{b}_{0}$ and $\left(L_{0}-\bar{L}_{0}\right)$, and apply the BRST charge $Q_{B}$ on it, the resulting state will not be annihilated by $b_{0}$ and $\bar{b}_{0}$. The part that is not annihilated by $b_{0}$ and $\bar{b}_{0}$ is given by $\left(c_{0}+\bar{c}_{0}\right) L_{0}|s\rangle=\frac{1}{4}\left(k^{2}+m^{2}\right)\left(c_{0}+\bar{c}_{0}\right)|s\rangle$. Hence the off-shell 'pure gauge' states will have to be defined as $Q_{B}|s\rangle-\frac{1}{4}\left(k^{2}+m^{2}\right)\left(c_{0}+\bar{c}_{0}\right)|s\rangle$. These are not annihilated by $Q_{B}$ but under the action of $Q_{B}$ give states proportional to $\left(k^{2}+m^{2}\right)$. Similarly physical states will now be defined by first continuing the momentum off-shell and then by adding appropriate linear combination of unphysical states proportional to $\left(k^{2}+m^{2}\right)$ so that they remain orthonormal to the pure gauge states. These will only be BRST invariant up to terms of order $\left(k^{2}+m^{2}\right)$ and transform under a conformal transformation as scalars up to terms of order $\left(k^{2}+m^{2}\right)$. Similar procedure can be used to define the unphysical states off-shell so that they remain orthogonal to physical states and themselves.

We shall denote by $|\alpha\rangle_{p},|s\rangle_{g}$ and $|s\rangle_{u}$ an appropriate basis of off-shell physical, pure gauge and unphysical states at mass level $m$, satisfying the identities

$$
{ }_{p}\langle\alpha \mid \beta\rangle_{p}=\delta_{\alpha \beta}, \quad{ }_{g}\langle r \mid s\rangle_{u}={ }_{u}\langle r \mid s\rangle_{g}=\delta_{r s}, \quad{ }_{p}\langle\alpha \mid s\rangle_{u}={ }_{p}\langle\alpha \mid s\rangle_{g}=0, \quad{ }_{g}\langle r \mid s\rangle_{g}={ }_{u}\langle r \mid s\rangle_{u}=0 .
$$

Note that this preserves the inner product matrix $\mathcal{I}$ given in (3.4). We shall see that at higher loop order we need to redefine the physical, unphysical and pure gauge states by making a further rotation of the basis. 


\section{$3.2 \quad$ Off-shell amplitudes}

In this subsection we shall describe the construction of off-shell amplitudes in string theory following [32], which in turn was inspired by bosonic string field theory [33] and other earlier work (e.g. [34, 35]). In order to define off-shell amplitudes in string theory we need to introduce local coordinate system around the punctures on the Riemann surface where the vertex operators are inserted [34] (see also [36-39]). Let us denote by $z$ a reference coordinate system on a Riemann surface, possibly consisting of several coordinate charts. Let $z_{i}$ denote the location of the $i$-th puncture in the $z$-coordinate system and $w_{i}$ denote the local coordinate system around the $i$-th puncture, related to $z$ by some functional relation $z=f_{i}\left(w_{i}\right)$ such that the $w_{i}=0$ point gets mapped to $z=z_{i}: f_{i}(0)=z_{i}$. Then the contribution to the $n$-point off-shell amplitude from the genus $g$ Riemann surfaces can be expressed as

$$
\int_{\mathcal{M}_{g ; n}}\left\langle\prod_{i=1}^{n} f_{i} \circ V_{i}(0) \times \text { ghost insertions }\right\rangle,
$$

where $f \circ V(0)$ denotes the conformal transformation of the vertex operator $V$ by the function $f(w)$, the correlator \langle\rangle is evaluated in the reference $z$-coordinate system and $\int_{\mathcal{M}_{g ; n}}$ denotes integration over the moduli space of Riemann surfaces of genus $g$ with $n$ punctures with appropriate measure. A detailed description of how to construct the integration measure (or equivalently the rules for inserting $b$-ghosts into the correlation function) for a given choice of local coordinate system can be found in [33, 34]. The offshell amplitudes defined this way depend on the choice of local coordinate system $w_{i}$ but are independent of the choice of the reference coordinate system $z$.

We shall work with a class of local coordinate systems satisfying the following properties: ${ }^{6}$

1. The local coordinate system is taken to be symmetric in all the puncture, 1.e. the function $f_{i}(w)$ should depend on $i$ only via the location $z_{i}$ of the puncture.

2. On 3-punctured sphere and 1-punctured tori the choice of the local coordinate system is arbitrary subject to condition 1 . We declare all 3-punctured spheres and 1-punctured tori to be one particle irreducible (1PI) contributions to genus zero 3point amplitudes and genus one 1-point amplitudes respectively.

3. We can construct a set of 4-punctured spheres by gluing a 3-punctured sphere with another 3-punctured sphere at one each of their punctures by the plumbing fixture procedure

$$
w_{1} w_{2}=e^{-s+i \theta} \quad 0 \leq \theta<2 \pi, \quad 0 \leq s<\infty .
$$

\footnotetext{
${ }^{6}$ We note that the choice of local coordinates which appear in the Siegel gauge amplitudes in closed bosonic string field theory of [33] automatically satisfies these requirements. Thus all our subsequent discussions hold for this theory. In particular our analysis shows that the renormalized physical masses are the same in different versions of closed string field theory using different vertices satisfying BatalinVilkovisky equations. Since these different versions are related to each other by field redefinitions together with a change in the gauge fixing condition [40] this indirectly tests gauge invariance of the renormalized physical masses in closed string field theory.
} 
Here $w_{1}$ and $w_{2}$ are the local coordinates at the punctures used for gluing. We choose the local coordinates on these 4-punctured spheres to be the ones induced from the local coordinates on the original 3-punctured spheres [41, 42], and declare the contribution from these 4-punctured spheres to off-shell four point amplitudes to be the one particle reducible (1PR) contributions to the genus zero four point amplitudes. On the rest of the genus zero four punctured Riemann surfaces we choose the local coordinate system arbitrarily subject to condition 1 and continuity and declare them to be 1PI contributions to genus zero four point amplitude. We shall use a shorthand notation calling the corresponding Riemann surfaces 1PI Riemann surfaces. Similarly by gluing a 3-punctured sphere to a 1-punctured torus we can generate a set of 2-punctured tori. We choose the local coordinates on these 2-punctured tori to be the ones induced from the local coordinates of the 3-punctured sphere and the 1-punctured torus, and declare their contribution to be the 1PR contribution to the genus one 2-point function. On the rest of the 2-punctured tori we choose the local coordinates arbitrarily subject to condition 1 and the requirement of continuity, and declare them to be 1PI contribution to the genus one 2-point amplitude.

4. We now repeat this process to Riemann surfaces of higher genus and/or higher number of punctures. At any stage, Riemann surfaces which can be obtained by gluing two or more 1PI Riemann surfaces to each other using the plumbing fixture procedure are declared to be contributions to 1PR amplitudes and on these Riemann surfaces the choice of local coordinates is induced from the local coordinates of the 1PI Riemann surfaces which have been glued. The rest of the Riemann surfaces are declared as 1PI contributions and the local coordinates at the punctures on these Riemann surfaces can be chosen arbitrarily subject to condition 1 and continuity.

We shall call the choice of local coordinates satisfying the criteria described above 'gluing compatible local coordinate system'. In the language of string field theory this has been called off-shell factorization, - a brief discussion and relevant references can be found in [43].

For our analysis it will also be useful to introduce the notion of amplitudes which are $1 P I$ in a given momentum $k$, where $k$ is the sum of a subset of the momenta carried by the external states of that amplitude. Riemann surfaces 1PI in the leg carrying momentum $k$ are defined to be those Riemann surfaces which cannot be obtained by gluing two or more 1PI or 1PR Riemann surfaces at punctures carrying momenta $k$ and $-k$. Thus this set of Riemann surfaces include the usual 1PI Riemann surfaces but also many 1PR Riemann surfaces which are obtained by gluing two or more 1PI Riemann surfaces at punctures carrying momenta other than $k$ or $-k$. The total contribution to an amplitude 1PI in momentum $k$ is then obtained by integrating over the moduli spaces of all Riemann surfaces which are 1PI in momentum $k$.

As an example consider genus one 2-point function with external vertex operators carrying momentum $k$ and $-k$. This receives contribution from 1PI Riemann surfaces and also 1PR Riemann surfaces obtained by gluing 1-punctured torus to 3-punctured sphere. 


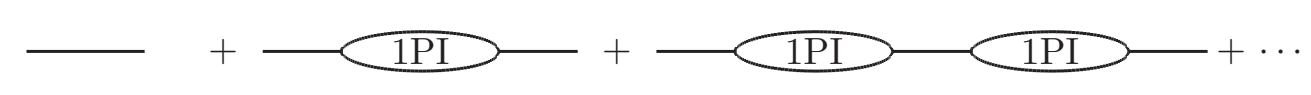

Figure 1. Pictorial representation of eq. (3.11).

However all of these are counted as 1PI in the momentum $k$ since the 1PR Riemann surfaces are obtained by gluing punctures carrying zero momentum, and not momentum $\pm k$.

\subsection{Off-shell amplitudes from 1PI amplitudes}

As we shall now discuss, the off-shell amplitudes constructed with the help of such choice of local coordinates can be organized in the same way that the full amplitudes in a quantum field theory can be organized as sums over tree level Feynman diagrams with 1PI amplitudes as vertices. As in [32] we begin our discussion with the propagator. We shall work with general off-shell string states of ghost number 2, as defined in section 3.1. If $\pm k$ denote the momenta carried by the external legs, then let $\widehat{\mathcal{F}}$ be the contribution to the off-shell two point amplitude from Riemann surfaces which are 1PI in momentum $k$. This includes sum over different genera starting from genus 1 . As discussed in [32], this can be regarded as a map from $\mathcal{H} \times \mathcal{H}$ to $\mathbb{C}$ where $\mathcal{H}$ denotes the Hilbert space of off-shell states of ghost number two as defined in section 3.1, but using the duality between ghost number two and ghost number four states by the BPZ inner product we can also regard this as a map from states of ghost number two to states of ghost number four which are annihilated by $c_{0}$ and $\bar{c}_{0}$. We can include a further action by $\bar{b}_{0} b_{0}$ to regard $\widehat{\mathcal{F}}$ as a map from $\mathcal{H}$ to $\mathcal{H}$. This is the viewpoint we shall adopt from now. The factor of $\bar{b}_{0} b_{0}$ in fact arises naturally in the tree level propagator of the string, which after being stripped of this factor, has the form

$$
\Delta=\frac{1}{4 \pi} \int_{0}^{\infty} d s \int_{0}^{2 \pi} d \theta e^{-s\left(L_{0}+\bar{L}_{0}\right)} e^{i \theta\left(L_{0}-\bar{L}_{0}\right)}=\frac{1}{2\left(L_{0}+\bar{L}_{0}\right)} \delta_{L_{0}, \bar{L}_{0}} .
$$

With this convention the full propagator is given by

$$
\Pi=\Delta+\Delta \widehat{\mathcal{F}} \Delta+\Delta \widehat{\mathcal{F}} \Delta \widehat{\mathcal{F}} \Delta+\cdots=\Delta(1-\widehat{\mathcal{F}} \Delta)^{-1}=(1-\Delta \widehat{\mathcal{F}})^{-1} \Delta .
$$

Pictorially this contribution can be represented as in figure 1 with the horizontal line denoting $\Delta$ and the blob marked 1PI denoting the contribution $\widehat{\mathcal{F}}$ from the Riemann surfaces that are 1PI in momentum $k$.

If $\mathcal{F}$ is the full off-shell two point function, then $\mathcal{F}$ and $\Pi$ are related by

$$
\Pi=\Delta+\Delta \mathcal{F} \Delta .
$$

Also $\mathcal{F}$ and $\widehat{\mathcal{F}}$ are related by

$$
\mathcal{F}=\widehat{\mathcal{F}}+\widehat{\mathcal{F}} \Delta \widehat{\mathcal{F}}+\cdots=\widehat{\mathcal{F}}(1-\Delta \widehat{\mathcal{F}})^{-1}=(1-\widehat{\mathcal{F}} \Delta)^{-1} \widehat{\mathcal{F}}=\widehat{\mathcal{F}}+\widehat{\mathcal{F}}\left(\Delta^{-1}-\widehat{\mathcal{F}}\right)^{-1} \widehat{\mathcal{F}} .
$$

Like $\widehat{\mathcal{F}}, \mathcal{F}, \Pi$ and $\Delta$ can be regarded as maps from $\mathcal{H}$ to $\mathcal{H}$.

As described in [32], we can use (3.13) to define $\widehat{\mathcal{F}}$ in terms of $\mathcal{F}$. At genus one $\widehat{\mathcal{F}}=\mathcal{F}$. Starting with this, we define $\widehat{\mathcal{F}}$ at genus two so as to satisfy (3.13) up to genus 


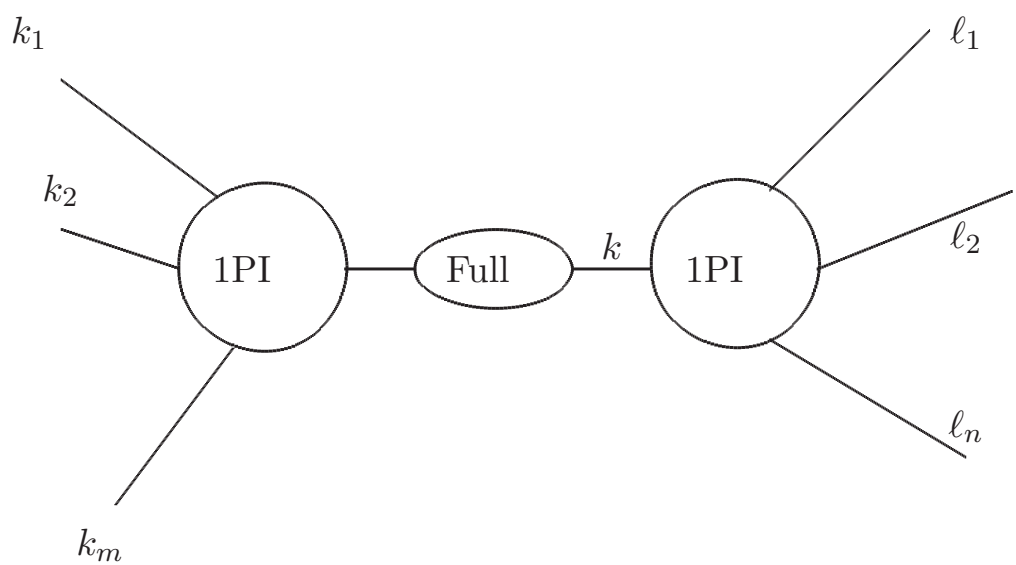

Figure 2. Pictorial representation of the second terms on the right hand sides of eq. (3.14). Here 1PI means sum of contributions which are 1PI in the leg carrying momentum $k$, whereas Full means sum of all contributions to the 2-point function shown in figure 1.

two. Physically the contribution to $\widehat{\mathcal{F}}$ at genus two is given by integrating over those Riemann surfaces which cannot be obtained by plumbing fixture of a pair of genus one Riemann surfaces. This definition of course depends on the choice of local coordinates at the punctures that we use to glue the two genus one Riemann surfaces. This procedure can be continued to define $\widehat{\mathcal{F}}$ at higher orders.

As another example let us consider an $m+n$ point amplitude $\Gamma$ with external momenta $k_{1}, \cdots k_{m}, \ell_{1}, \cdots \ell_{n}$ satisfying $\sum_{i=1}^{m} k_{i}=-\sum_{j=1}^{n} \ell_{j}=k$, and other quantum numbers $a_{1}, \cdots a_{m}, b_{1}, \cdots b_{n}$. Our goal is to express the amplitude in a way that makes manifest the poles in the momentum $k$. For this we introduce two auxiliary quantities: $\Gamma_{1}^{a}$ describing the contribution to $(m+1)$-point functions with external states carrying quantum numbers $a_{1}, \cdots a_{m}, a$ and momenta $k_{1}, \cdots k_{m},-k$ and $\Gamma_{2}^{b}$ describing the contribution to $(n+1)$ point functions with external states carrying quantum numbers $b_{1}, \cdots b_{n}, b$ and momenta $\ell_{1}, \cdots \ell_{n}, k$. Here the quantum numbers $a$ and $b$ run over all off-shell string states of ghost number 2. Note that we have not explicitly exhibited the dependence of $\Gamma_{1}$ on the indices $a_{1}, \cdots a_{m}$ and momenta $k_{1}, \cdots k_{m}$ for brevity; a similar comment holds for $\Gamma_{2}$. We shall also introduce the quantities $\widehat{\Gamma}_{1}^{a}$ and $\widehat{\Gamma}_{2}^{b}$ which describe contributions to $\Gamma_{1}^{a}$ and $\Gamma_{2}^{b}$ from those Riemann surfaces which are 1PI in the leg carrying momentum $k$ (in the sense described at the end of section 3.2). Then the full contribution to $\Gamma$ can be expressed as

$$
\begin{aligned}
\Gamma & =\widehat{\Gamma}+\widehat{\Gamma}_{1}^{a} \mathcal{I}_{a c} \Pi_{c b} \widehat{\Gamma}_{2}^{b} \\
& =\widehat{\Gamma}+\widehat{\Gamma}_{1}^{T} \mathcal{I} \Delta(1-\widehat{\mathcal{F}} \Delta)^{-1} \widehat{\Gamma}_{2} \\
& =\widehat{\Gamma}+\widehat{\Gamma}_{1}^{T} \mathcal{I}(1-\Delta \widehat{\mathcal{F}})^{-1} \Delta \widehat{\Gamma}_{2},
\end{aligned}
$$

where $\widehat{\Gamma}$ represents contributions to $\Gamma$ which are $1 \mathrm{PI}$ in the leg carrying momentum $k$ and $\mathcal{I}$ is the inner product matrix (3.4) over the full space of off-shell string states. The equality between different expressions on the right hand sides of (3.14) follows from (3.11). A pictorial representation of the second term on the right hand side of the first line of (3.14) has been shown in figure 2 


\section{Physical state propagator in string theory}

In this section we shall generalize the gauge theory analysis of section 2 to give an iterative procedure for constructing physical state propagator in string theory. From this we can compute the masses of physical states.

\subsection{Renormalized propagator at a given mass level}

Since string theory contains infinite number of states, the quantities $\Pi, \Delta, \mathcal{F}$ and $\widehat{\mathcal{F}}$ introduced in section 3.3 are all infinite dimensional matrices. Our first step will be to 'integrate out' all states except the ones at mass level $m$ so that we can work with finite dimensional matrices with rows and columns labelled by states at mass level $m .^{7}$ For this we denote by $P_{T}$ the total projection operator at mass level $m$,

$$
P_{T}=\left\{|\alpha\rangle_{p p}\langle\alpha|+| s\rangle_{g u}\langle s|+| s\rangle_{u}\langle s|\right\}
$$

and define

$$
\begin{gathered}
\bar{\Delta}=\Delta-\left(k^{2}+m^{2}\right)^{-1} P_{T}, \\
\overline{\mathcal{F}}=\widehat{\mathcal{F}}+\widehat{\mathcal{F}} \bar{\Delta} \widehat{\mathcal{F}}+\cdots=\widehat{\mathcal{F}}(1-\bar{\Delta} \widehat{\mathcal{F}})^{-1}=(1-\widehat{\mathcal{F}} \bar{\Delta})^{-1} \widehat{\mathcal{F}}=\widehat{\mathcal{F}}+\widehat{\mathcal{F}}\left(\bar{\Delta}^{-1}-\widehat{\mathcal{F}}\right)^{-1} \widehat{\mathcal{F}},
\end{gathered}
$$

where $\widehat{\mathcal{F}}$ has been defined in section 3.3. It is clear from the definition of $\bar{\Delta}$ and $\widehat{\mathcal{F}}$ that their genus expansions do not have any poles at $k^{2}=-m^{2}$. Hence $\overline{\mathcal{F}}$ defined in (4.3) also does not have such poles. From (3.13), (4.3) we get

$$
\mathcal{F}=\overline{\mathcal{F}}\left\{1-\left(k^{2}+m^{2}\right)^{-1} P_{T} \overline{\mathcal{F}}\right\}^{-1}=\left\{1-\overline{\mathcal{F}}\left(k^{2}+m^{2}\right)^{-1} P_{T}\right\}^{-1} \overline{\mathcal{F}} .
$$

We now define

$$
\mathcal{V}_{T}=P_{T} \mathcal{I} \Pi P_{T}, \quad \widetilde{F}_{T}=P_{T} \mathcal{I} \overline{\mathcal{F}} P_{T}, \quad F_{T}=P_{T} \mathcal{I} \mathcal{F} P_{T},
$$

where $\Pi$ has been defined in (3.11). Physically $F_{T}$ denotes the two point amplitude restricted to external states of mass level $m, \widetilde{F}_{T}$ is the contribution to $F_{T}$ that is 1PI in momentum $k$ after integrating out all states other than those at mass level $m$, and $\mathcal{V}_{T}$ denotes the off-shell two point Green's function restricted to external states of mass level $m$. It follows from (4.4), (4.5) that

$$
\begin{aligned}
& F_{T}=\widetilde{F}_{T}\left(1-\left(k^{2}+m^{2}\right)^{-1} \mathcal{I} \widetilde{F}_{T}\right)^{-1}, \\
& \mathcal{V}_{T}=\left(k^{2}+m^{2}\right)^{-1} \mathcal{I} P_{T}+\left(k^{2}+m^{2}\right)^{-2} F_{T}=P_{T}\left\{\left(k^{2}+m^{2}\right) \mathcal{I}-\widetilde{F}_{T}\right\}^{-1},
\end{aligned}
$$

where it is understood that the inverse on the right hand sides is being taken in the finite dimensional subspace of mass level $m$ states only. We shall label the matrices $\widetilde{F}_{T}$ and $\mathcal{V}_{T}$ as

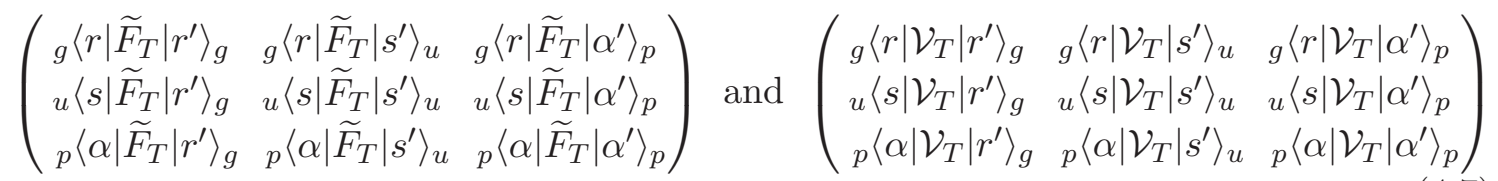

\footnotetext{
${ }^{7}$ Throughout this paper we shall denote by states at mass level $m$ all states which have tree level mass $m$, 1.e. states which are annihilated by $L_{0}$ and $\bar{L}_{0}$ when $k^{2}=-m^{2}$.
} 
respectively.

$\mathcal{V}_{T}$ and $\widetilde{F}_{T}$ and the inner product matrix $\mathcal{I}$ are the exact analogs of the corresponding quantities defined in section 2. In particular the genus expansion of $\widetilde{F}_{T}$ is free from any poles at $k^{2}=-m^{2}$ at every order. In section 4.3 we shall generalize the procedure of section 2 to construct the propagator of physical states.

One point worth emphasizing is that for our analysis we do not really need the gluing compatibility condition discussed in section 3.2 to be valid for the whole range $0 \leq s<$ $\infty$ with $s$ defined in (3.9); it is sufficient if the compatibility condition holds in a small neighborhood of degeneration points, e.g. for $s \geq s_{0}$ for some constant $s_{0}$. One way to see this is that we can rescale all the local coordinates $w_{i}$ to bring the range $s \geq s_{0}$ in (3.9) to $s \geq 0$. This will have the effect of rescaling all the off-shell amplitudes by some power of $e^{-s_{0}\left(k^{2}+m^{2}\right)}$. But we can also proceed with the original choice of local coordinates and repeat the whole analysis by changing the definition of $1 \mathrm{PI}$ and $1 \mathrm{PR}$ amplitudes so that two or more 1PI amplitudes glued together using (3.9) for $s \geq s_{0}$ are now declared as 1PR. We also have to modify the definition of $\Delta$ given in (3.10), with the integral over $s$ now running from $s_{0}$ to $\infty$. This will produce a multiplicative factor of $e^{-s_{0}\left(L_{0}+\bar{L}_{0}\right)}$ in the definition of $\Delta$. But the rest of the analysis is not affected by this. In particular we can continue to define $\bar{\Delta}$ and $\overline{\mathcal{F}}$ via eqs. (4.2) and (4.3). The contribution to $\bar{\Delta}$ from states of mass level $m$ now gives $P_{T}\left(k^{2}+m^{2}\right)^{-1}\left(e^{-s_{0}\left(k^{2}+m^{2}\right) / 2}-1\right)$. Since this does not have a pole at $\left(k^{2}+m^{2}\right)=0, \bar{\Delta}$ and $\overline{\mathcal{F}}$ will continue to be free from poles at $k^{2}+m^{2}=0$.

\subsection{An alternate definition of $\widetilde{F}_{T}$}

The definition of $\widetilde{F}_{T}$ given in section 4.1 looks complicated, since we first need to define the 1PI amplitudes $\widehat{\mathcal{F}}$, then construct $\overline{\mathcal{F}}$ via (4.3) and finally project onto the mass level $m$ sector as in (4.5). In particular the definition of $\widehat{\mathcal{F}}$ requires dividing up the moduli space of Riemann surfaces into 1PI and 1PR parts. Since $\widetilde{F}_{T}$ will play a crucial role in the definition of the physical renormalized mass, we shall now give an alternate definition of $\widetilde{F}_{T}$ which does not require us to explicitly identify the 1PI subspace in the moduli space of Riemann surfaces. For this we note from (4.6) that

$$
\begin{aligned}
\widetilde{F}_{T} & =F_{T}\left(1+\left(k^{2}+m^{2}\right)^{-1} \mathcal{I} F_{T}\right)^{-1} \\
& =F_{T}-F_{T} \mathcal{I}\left(k^{2}+m^{2}\right)^{-1} F_{T}+F_{T} \mathcal{I}\left(k^{2}+m^{2}\right)^{-1} F_{T} \mathcal{I}\left(k^{2}+m^{2}\right)^{-1} F_{T}+\cdots .
\end{aligned}
$$

Now $F_{T}$ has a simple interpretation since it denotes the full off-shell 2-point function restricted to mass level $m$. Thus we can regard (4.8) as the definition of $\widetilde{F}_{T}$. In this way of defining $\widetilde{F}_{T}$ we never have to divide the contribution to an amplitude into 1PI and 1PR parts. The only price we pay is that from (4.8) it is not obvious that $\widetilde{F}_{T}$ is free from poles at $k^{2}+m^{2}=0$, since each term on the right hand side of (4.8) does contain such poles. Nevertheless our previous arguments guarantee that all such poles cancel.

It may seem that $\widetilde{F}_{T}$ defined this way requires less information than in the earlier definition, but this is not the case. The definition of $\widetilde{F}_{T}$ requires information on the choice of local coordinate system, which in turn completely fixes the division of the amplitudes into $1 \mathrm{PI}$ and $1 \mathrm{PR}$ parts. Thus even though we do not explicitly use this division in defining 
$\widetilde{F}_{T}$, the data used in the construction of $\widetilde{F}_{T}$ is sufficient to determine the division of an amplitude into 1PI and 1PR parts.

The definition of $\widetilde{F}_{T}$ given in this subsection will be useful when we generalize the analysis to super and heterotic string theories.

\subsection{Renormalized physical state propagator and masses}

Following the analysis of section 2.2 we now seek a change of basis

$$
|\alpha\rangle_{p}^{\prime}=A_{\beta \alpha}|\beta\rangle_{p}+B_{s \alpha}|s\rangle_{g}+C_{s \alpha}|s\rangle_{u}, \quad|r\rangle_{g}^{\prime}=|r\rangle_{g}+D_{\beta r}|\beta\rangle_{p}, \quad|r\rangle_{u}^{\prime}=|r\rangle_{u}+K_{\beta r}|\beta\rangle_{p},
$$

such that the following conditions hold

$$
{ }_{p}^{\prime}\langle\alpha \mid \beta\rangle_{p}^{\prime}=\delta_{\alpha \beta}, \quad{ }_{p}\langle\alpha \mid s\rangle_{u}^{\prime}={ }_{p}{ }_{p}\langle\alpha \mid s\rangle_{g}^{\prime}={ }_{u}\langle r \mid \beta\rangle_{p}^{\prime}={ }_{g}\langle r \mid \beta\rangle_{p}^{\prime}=0,
$$

and

$$
{ }_{p}^{\prime}\left\langle\alpha\left|\widetilde{F}_{T}\right| s\right\rangle_{u}^{\prime}={ }_{p}{ }_{p}\left\langle\alpha\left|\widetilde{F}_{T}\right| s\right\rangle_{g}^{\prime}={ }_{u}{ }_{u}\left\langle r\left|\widetilde{F}_{T}\right| \beta\right\rangle_{p}^{\prime}={ }_{g}\left\langle r\left|\widetilde{F}_{T}\right| \beta\right\rangle_{p}^{\prime}=0 .
$$

We now substitute (4.9) into (4.10), (4.11) and use (3.7) to get ${ }^{8}$

$$
\begin{gathered}
\left(A^{\dagger} A+B^{\dagger} C+C^{\dagger} B\right)_{\alpha \beta}=\delta_{\alpha \beta}, \quad\left(D^{\dagger} A+C\right)_{r \alpha}=0, \quad\left(K^{\dagger} A+B\right)_{r \alpha}=0, \\
{ }_{u}\left\langle r\left|\widetilde{F}_{T}\right| \alpha\right\rangle_{p} A_{\alpha \beta}+{ }_{u}\left\langle r\left|\widetilde{F}_{T}\right| s\right\rangle_{g} B_{s \beta}+{ }_{u}\left\langle r\left|\widetilde{F}_{T}\right| s\right\rangle_{u} C_{s \beta}+\left(K^{\dagger}\right)_{r \gamma p}\left\langle\gamma\left|\widetilde{F}_{T}\right| \alpha\right\rangle_{p} A_{\alpha \beta} \\
+\left(K^{\dagger}\right)_{r \gamma p}\left\langle\gamma\left|\widetilde{F}_{T}\right| s\right\rangle_{g} B_{s \beta}+\left(K^{\dagger}\right)_{r \gamma p}\left\langle\gamma\left|\widetilde{F}_{T}\right| s\right\rangle_{u} C_{s \beta}=0 \\
{ }_{g}\left\langle r\left|\widetilde{F}_{T}\right| \alpha\right\rangle_{p} A_{\alpha \beta}+{ }_{g}\left\langle r\left|\widetilde{F}_{T}\right| s\right\rangle_{g} B_{s \beta}+{ }_{g}\left\langle r\left|\widetilde{F}_{T}\right| s\right\rangle_{u} C_{s \beta}+\left(D^{\dagger}\right)_{r \gamma p}\left\langle\gamma\left|\widetilde{F}_{T}\right| \alpha\right\rangle_{p} A_{\alpha \beta} \\
+\left(D^{\dagger}\right)_{r \gamma p}\left\langle\gamma\left|\widetilde{F}_{T}\right| s\right\rangle_{g} B_{s \beta}+\left(D^{\dagger}\right)_{r \gamma p}\left\langle\gamma\left|\widetilde{F}_{T}\right| s\right\rangle_{u} C_{s \beta}=0 .
\end{gathered}
$$

Let us first count the number of independent variables and the number of independent equations. The number of real components in the variables $A_{\alpha \beta}, B_{s \alpha}, C_{s \alpha}, D_{\beta r}$ and $K_{\beta r}$ are

$$
2 n_{p}^{2}+4 \times 2 n_{p} n_{g}
$$

where $n_{p}$ is the number of physical states and $n_{g}=n_{u}$ is the number of pure gauge / unphysical states at mass level $m$. On the other hand the number of independent equations can be counted as follows. Since both sides of the first equation in (4.12) are hermitian matrices, this gives $n_{p}^{2}$ real equations, whereas each of the rest gives $2 n_{p} n_{g}$ real equations. Thus the total number of equations is

$$
n_{p}^{2}+4 \times 2 n_{p} n_{g}
$$

Thus we see that we have $n_{p}^{2}$ extra variables compared to the number of equations. This can be traced to the freedom of multiplying $A, B$ and $C$ by a unitary matrix from the right which is a symmetry of the equations (4.12) (and represent the freedom of a unitary rotation in the subspace of physical states $\left.|\alpha\rangle_{p}^{\prime}\right)$. Up to this freedom we can determine the matrices $A, \cdots K$ by solving (4.12).

\footnotetext{
${ }^{8}$ We seek a change of basis that is real in the position space. In momentum space this implies that changing the momentum from $k$ to $-k$ has the effect of complex conjugating the coefficients $A_{\alpha \beta}, \cdots K_{\beta r}$. This has been used in (4.12).
} 
We shall now describe an iterative procedure for solving these equations. For this we note that the leading (genus one) contribution to $\widetilde{F}_{T}$ satisfies the property

$$
{ }_{p}\left\langle\alpha\left|\widetilde{F}_{T}\right| s\right\rangle_{g} \sim \lambda\left(k^{2}+m^{2}\right), \quad{ }_{g}\left\langle r\left|\widetilde{F}_{T}\right| s\right\rangle_{g} \sim \lambda\left(k^{2}+m^{2}\right), \quad{ }_{g}\left\langle r\left|\widetilde{F}_{T}\right| \beta\right\rangle_{p} \sim \lambda\left(k^{2}+m^{2}\right),
$$

where $\lambda$ now stands for the genus expansion parameter given by the square of the string coupling. These properties follow from the fact that at genus one $\widetilde{F}_{T}$ includes the full contribution to the torus two point function. Representing a pure gauge state as $Q_{B}|n\rangle$ plus a term of order $\left(k^{2}+m^{2}\right)$, deforming the contour of integration of the BRST current so that it acts on the other vertex operator, and then using that fact that acting on an off-shell physical or pure gauge state $Q_{B}$ gives a term proportional to $\left(k^{2}+m^{2}\right)$, we arrive at (4.15). This in turn allows us to look for solutions where at order $\lambda^{0}$,

$$
A, B, K \sim 1, \quad C, D \sim\left(k^{2}+m^{2}\right) .
$$

The solution to order $\lambda^{0}$ and leading order in $k^{2}+m^{2}$ are given by

$$
\begin{aligned}
A_{\alpha \beta} & =\delta_{\alpha \beta}+\mathcal{O}\left(k^{2}+m^{2}\right), \\
C & =-D^{\dagger}+\mathcal{O}\left(k^{2}+m^{2}\right), \\
B & =-K^{\dagger}+\mathcal{O}\left(k^{2}+m^{2}\right), \\
\lambda^{-1}\left\{\delta_{\beta \gamma u}\left\langle r\left|\widetilde{F}_{T}\right| s\right\rangle_{g}-\delta_{r s p}\left\langle\gamma\left|\widetilde{F}_{T}\right| \beta\right\rangle_{p}\right\} K_{s \gamma}^{\dagger} & =\lambda^{-1}\left\langle{ }_{u}\left\langle\widetilde{F}_{T} \mid \beta\right\rangle_{p}+\mathcal{O}\left(k^{2}+m^{2}\right)\right. \\
\lambda^{-1}\left\{\delta_{\beta \gamma g}\left\langle r\left|\widetilde{F}_{T}\right| s\right\rangle_{u}-\delta_{r s p}\left\langle\gamma\left|\widetilde{F}_{T}\right| \beta\right\rangle_{p}\right\} D_{s \gamma}^{\dagger} & =\lambda_{g}^{-1}\left\langle r\left|\widetilde{F}_{T}\right| \beta\right\rangle_{p}+\lambda^{-1}{ }_{g}\left\langle r\left|\widetilde{F}_{T}\right| s\right\rangle_{g} B_{s \beta}+\mathcal{O}\left(\left(k^{2}+m^{2}\right)^{2}\right) .
\end{aligned}
$$

This gives a sensible solution satisfying (4.16) provided the $n_{p} n_{g} \times n_{p} n_{g}$ matrix

$$
S_{r \beta, s \gamma} \equiv \lambda^{-1}\left\{{ }_{u}\left\langle r\left|\widetilde{F}_{T}\right| s\right\rangle_{g} \delta_{\beta \gamma}-{ }_{p}\left\langle\gamma\left|\widetilde{F}_{T}\right| \beta\right\rangle_{p} \delta_{r s}\right\},
$$

is invertible. Starting with this solution we can solve for the matrices $A, B, C, D, K$ iteratively in powers of the genus expansion parameter $\lambda$ and $\left(k^{2}+m^{2}\right)$ exactly as in section 2 . As long as the matrix defined in (4.18) is invertible, the coefficient of $\lambda^{n}$ for any $n$ is free from poles near $k^{2}=-m^{2}$. Physically, invertibility of $S_{r \beta, s \gamma}$ is the condition that the degeneracy between the masses of physical states and the unphysical / pure gauge states is lifted at one loop order. If this condition fails then we need to go to higher order in perturbation theory to lift the degeneracy. We expect that in principle there should be no difficulty in carrying out this procedure, although in practice the analysis is likely to become more complicated.

The coefficients $A, \cdots K$ satisfying (4.12) ensures, via eqs. (4.9)-(4.11) that the matrices $\mathcal{I}$ and $\widetilde{F}_{T}$ expressed in the primed basis have block diagonal form, with no cross terms between the states $|\alpha\rangle_{p}^{\prime}$ and $\left(|r\rangle_{u}^{\prime},|r\rangle_{g}^{\prime}\right)$. As in section 2 we define

$$
\widetilde{F}_{\alpha \beta}(k)={ }_{p}^{\prime}\left\langle\alpha\left|\widetilde{F}_{T}\right| \beta\right\rangle_{p}^{\prime} .
$$

Then the propagator restricted to the modified physical sector is given by

$$
\mathcal{V}_{\alpha \beta} \equiv{ }_{p}^{\prime}\left\langle\alpha\left|\mathcal{V}_{T}\right| \beta\right\rangle_{p}^{\prime}=\left(\left(k^{2}+m^{2}-\widetilde{F}(k)\right)^{-1}\right)_{\alpha \beta} .
$$


From here onwards we proceed as in [32]. We can diagonalize $\widetilde{F}(k)$ as

$$
\widetilde{F}(k)=U(k) \widetilde{F}_{d}(k) U(k)^{\dagger}, \quad U(k)^{\dagger}=U(k)^{-1}=U(-k)^{T},
$$

so that we have

$$
\mathcal{V}=U(k)\left(k^{2}+m^{2}-\widetilde{F}_{d}(k)\right)^{-1} U(k)^{\dagger} .
$$

We can now determine the solutions to the equation $k^{2}+m^{2}-\widetilde{F}_{d}(k)=0$ iteratively for each of the diagonal entries of $\widetilde{F}_{d}(k)$, starting with $k^{2}=-m^{2}$ as the leading order solution. This gives the physical masses. Let $M_{p}^{2}$ denotes the diagonal matrix with the diagonal elements being equal to the squares of the physical masses. Then we can express $\left(k^{2}+m^{2}-\widetilde{F}_{d}(k)\right)^{-1}$ as

$$
X_{d}(k)\left(k^{2}+M_{p}^{2}\right)^{-1}
$$

where $X_{d}(k)$ is a diagonal matrix which has no poles near $k^{2}=-m^{2}$. Eq. (4.22) now allows us to express the physical propagator $\mathcal{V}_{\alpha \beta}$ as

$$
\mathcal{V}=Z^{1 / 2}(k)\left(k^{2}+M_{p}^{2}\right)^{-1} Z^{1 / 2}(-k)^{T}, \quad Z^{1 / 2}(k) \equiv U(k) X_{d}(k)^{1 / 2} .
$$

In section 6 we shall argue that the squares of the physical masses given by the diagonal elements of $M_{p}^{2}$ do not depend on the choice of local coordinates at the punctures, although the wave-function renormalization matrix $Z^{1 / 2}(k)$ does depend on the choice of local coordinates.

Finally we would like to note that since $B_{s \alpha}$ is of order unity, the corrected physical state $|\alpha\rangle_{p}^{\prime}$ differs from the tree level physical state $|\alpha\rangle_{p}$ by a pure gauge state with coefficient of order unity. Thus even in the $\lambda \rightarrow 0$ limit, $|\alpha\rangle_{p}^{\prime}$ does not approach $|\alpha\rangle_{p}$.

\subsection{Renormalized masses in the unphysical / pure gauge sector}

We shall now briefly describe the computation of the renormalized masses in the unphysical / pure gauge sector by generalizing the procedure described in section 2.4. For this we define

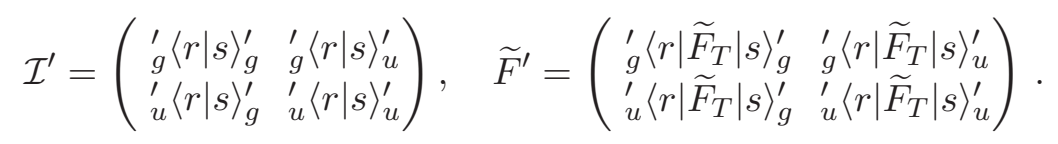

Then the renormalized mass ${ }^{2}$ 's in the unphysical / pure gauge sector will be given by the zeroes of the eigenvalues of the matrix

$$
\left(k^{2}+m^{2}\right) \mathcal{I}^{\prime}-\widetilde{F}^{\prime}(k),
$$

in the complex $-k^{2}$ plane. To evaluate the order $\lambda$ correction to these masses, we shall assume as in section 2.4 that $k^{2}+m^{2}$ is of order $\lambda$ when $-k^{2}$ is equal to the renormalized mass $^{2}$ and keep terms in (4.26) up to order $\lambda$. Using (4.9), (4.15) and (4.17) one finds that to order unity

$$
{ }_{g}^{\prime}\langle r \mid s\rangle_{g}^{\prime}=0, \quad{ }_{g}^{\prime}\langle r \mid s\rangle_{u}^{\prime}={ }_{u}^{\prime}\langle r \mid s\rangle_{g}^{\prime}=\delta_{r s}
$$

and to order $\lambda$,

$$
{ }_{g}^{\prime}\left\langle r\left|\widetilde{F}_{T}\right| s\right\rangle_{g}^{\prime}=0, \quad{ }_{g}^{\prime}\left\langle r\left|\widetilde{F}_{T}\right| s\right\rangle_{u}^{\prime}={ }_{g}\left\langle r\left|\widetilde{F}_{T}\right| s\right\rangle_{u}, \quad{ }_{u}\left\langle r\left|\widetilde{F}_{T}\right| s\right\rangle_{g}^{\prime}={ }_{u}\left\langle r\left|\widetilde{F}_{T}\right| s\right\rangle_{g} .
$$


Hence to order $\lambda$ (counting $k^{2}+m^{2}$ as order $\lambda$ )

$$
\left(k^{2}+m^{2}\right) \mathcal{I}^{\prime}-\widetilde{F}^{\prime}(k)=\left(\begin{array}{cr}
0 & \left(k^{2}+m^{2}\right) \delta_{r s}-{ }_{g}\left\langle r\left|\widetilde{F}_{T}\right| s\right\rangle_{u} \\
\left(k^{2}+m^{2}\right) \delta_{r s}-{ }_{u}\left\langle r\left|\widetilde{F}_{T}\right| s\right\rangle_{g} & \left(k^{2}+m^{2}\right){ }_{u}^{\prime}\langle r \mid s\rangle_{u}^{\prime}-{ }_{u}^{\prime}\left\langle r\left|\widetilde{F}_{T}\right| s\right\rangle_{u}^{\prime}
\end{array}\right) .
$$

Using the fact that the vanishing of an eigenvalue of a matrix is equivalent to requiring the vanishing of its determinant, we see that the required condition is the vanishing of the determinant of the upper right (or lower left) block. This in turn is equivalent to requiring the vanishing of an eigenvalue of

$$
\left(k^{2}+m^{2}\right) \delta_{r s}-{ }_{g}\left\langle r\left|\widetilde{F}_{T}\right| s\right\rangle_{u}
$$

as a function of $-k^{2}$. Starting with this first order solution one can iteratively compute higher order corrections to the renormalized mass ${ }^{2}$ in the unphysical / pure gauge sector by looking for zero eigenvalues of (4.26).

\subsection{Dependence on choice of local coordinates}

An important question is: how do the physical masses depend on the choice of local coordinates? We shall postpone a full discussion on this till section 6 , but at this stage we can derive the result at order $\lambda$. The locations of the physical mass squares are determined by the zeroes of $k^{2}+m^{2}-\widetilde{F}_{d}(k)$ in the $-k^{2}$ plane. Let us focus on the one loop, 1.e. order $\lambda$ correction to the mass ${ }^{2}$. For this we need to determine the function $\widetilde{F}_{d}(k)$ and hence $\widetilde{F}(k)$ to order $\lambda$ at $k^{2}=-m^{2}$. It follows from (4.9), (4.15), (4.17), (4.19) and the fact that the leading contribution to $\widetilde{F}_{T}$ is of order $\lambda$ that to order $\lambda$ and at $k^{2}+m^{2}=0$

$$
\widetilde{F}_{\alpha \beta}=\left\langle\alpha\left|\widetilde{F}_{T}\right| \beta\right\rangle \text {. }
$$

At order $\lambda$ this represents the full two point function of the tree level physical states $|\alpha\rangle$ and $|\beta\rangle$ on the torus. Since $|\alpha\rangle$ and $|\beta\rangle$ are both dimension zero primaries at $k^{2}=-m^{2}$, we see that to this order $\widetilde{F}_{\alpha \beta}$ at $k^{2}=-m^{2}$ is independent of the choice of local coordinates. Hence the renormalized physical masses are also independent of the choice of local coordinates to this order.

We can also consider the fate of the masses in the unphysical / pure gauge sector under a change in the local coordinate system. To order $\lambda$ the mass ${ }^{2}$ 's in this sector are given by the zeroes of the eigenvalues of the matrix (4.30) in the $-k^{2}$ plane. Since the matrix elements ${ }_{g}\left\langle r\left|\widetilde{F}_{T}\right| s\right\rangle_{u}$ involve unphysical and pure gauge states, which are generically not dimension zero primaries, we see that in the generic case the order $\lambda$ contribution to the masses of the unphysical and pure gauge states will depend on the choice of local coordinates. ${ }^{9}$ Higher order contributions can correct these results but cannot cancel the order $\lambda$ corrections. This we conclude that the unphysical / pure gauge sector masses do depend on the choice of local coordinate system.

\footnotetext{
${ }^{9}$ If the vertex operator involves ghost excitations then the integration measure provided by $b$-ghost insertions also depend on the choice of local coordinates [33, 34].
} 


\section{Poles of S-matrix elements of massless / BPS / special states}

In this section we shall show that if we consider an S-matrix of external massless, BPS and/or special states then the poles in this S-matrix in any channel are the same ones as those which appear in the analysis of section $4.3 .{ }^{10}$ Let us denote by $k$ the total momentum carried in some particular internal channel, being equal to the sum of momenta of two or more external states, and look for poles in the $-k^{2}$ plane. Our starting point will be the expression (3.14) for the $(m+n)$-point amplitude. The S-matrix elements are obtained from this by multiplying this by appropriate renormalization factors on the external legs and then setting the external momenta on-shell. Since multiplicative factors on the external legs do not affect the locations of the poles in the $k^{2}$ plane, we can directly use $\Gamma$ to examine these poles. Our interest will be to look for those poles which arise from states at mass level $m$. For this it will be useful to 'integrate out' the states at other mass levels. With this goal in mind, we define

$$
\begin{aligned}
\bar{\Gamma}_{1}^{T} \mathcal{I} & =\widehat{\Gamma}_{1}^{T} \mathcal{I}(1+\bar{\Delta} \widehat{\mathcal{F}}+\bar{\Delta} \widehat{\mathcal{F}} \bar{\Delta} \widehat{\mathcal{F}}+\cdots)=\widehat{\Gamma}_{1}^{T} \mathcal{I}(1-\bar{\Delta} \widehat{\mathcal{F}})^{-1}, \\
\bar{\Gamma}_{2} & =(1+\widehat{\mathcal{F}} \bar{\Delta}+\widehat{\mathcal{F}} \bar{\Delta} \widehat{\mathcal{F}} \bar{\Delta}+\cdots) \widehat{\Gamma}_{2}=(1-\widehat{\mathcal{F}} \bar{\Delta})^{-1} \widehat{\Gamma}_{2},
\end{aligned}
$$

where $\bar{\Delta}$ has been defined in (4.2). We also define

$\bar{\Gamma}=\widehat{\Gamma}+\widehat{\Gamma}_{1}^{T} \mathcal{I}(\bar{\Delta}+\bar{\Delta} \widehat{\mathcal{F}} \bar{\Delta}+\bar{\Delta} \widehat{\mathcal{F}} \bar{\Delta} \widehat{\mathcal{F}} \bar{\Delta}+\cdots) \widehat{\Gamma}_{2}=\widehat{\Gamma}+\widehat{\Gamma}_{1}^{T} \mathcal{I} \bar{\Delta}(1-\widehat{\mathcal{F}} \bar{\Delta})^{-1} \widehat{\Gamma}_{2}=\widehat{\Gamma}+\widehat{\Gamma}_{1}^{T} \mathcal{I}(1-\bar{\Delta} \widehat{\mathcal{F}})^{-1} \bar{\Delta} \widehat{\Gamma}_{2}$.

Using (3.14), (4.2), (4.3), (5.1) and (5.2) we now get

$$
\begin{aligned}
\Gamma & =\bar{\Gamma}+\bar{\Gamma}_{1}^{T} \mathcal{I}\left\{1-\left(k^{2}+m^{2}\right)^{-1} P_{T} \overline{\mathcal{F}}\right\}^{-1} P_{T}\left(k^{2}+m^{2}\right)^{-1} \bar{\Gamma}_{2} \\
& =\bar{\Gamma}+\bar{\Gamma}_{1}^{T} \mathcal{I} P_{T}\left(k^{2}+m^{2}\right)^{-1}\left\{1-\left(k^{2}+m^{2}\right)^{-1} \overline{\mathcal{F}} P_{T}\right\}^{-1} \bar{\Gamma}_{2} \\
& =\bar{\Gamma}+\bar{\Gamma}_{1}^{T} \mathcal{I} P_{T}\left(k^{2}+m^{2}-P_{T} \overline{\mathcal{F}} P_{T}\right)^{-1} P_{T} \bar{\Gamma}_{2} \\
& =\bar{\Gamma}+\bar{\Gamma}_{1}^{T} \mathcal{V}_{T} \bar{\Gamma}_{2},
\end{aligned}
$$

where $\mathcal{V}_{T}$ has been defined in (4.5). Now the genus expansions of $\bar{\Gamma}, \bar{\Gamma}_{1}^{T}$ and $\bar{\Gamma}_{2}$ are free from poles at $-k^{2}=m^{2}$. Thus the only poles near $-k^{2}=m^{2}$ can come from the poles of matrix $\mathcal{V}_{T}$. These are precisely the renormalized physical and unphysical squared masses as discussed in section 4 .

For later use, it will be useful to isolate the contribution from the physical states from that of the unphysical and pure gauge states. For this we insert the projection operator $P_{T}$ on both sides of $\mathcal{V}_{T}$ on the right hand side of (5.3) using the identity $P_{T} \mathcal{V}_{T} P_{T}=\mathcal{V}_{T}$. Now using (4.1) and (4.9), $P_{T}$ may be expressed as

$$
P_{T}=\sum_{\alpha}|\alpha\rangle_{p}^{\prime}{ }_{p}^{\prime}\langle\alpha|+\sum_{r, s}\left[\widetilde{A}_{r s}|r\rangle_{g}^{\prime}{ }_{g}^{\prime}\left\langle s\left|+\widetilde{B}_{r s}\right| r\right\rangle_{g}^{\prime}{ }_{u}^{\prime}\left\langle s\left|+\widetilde{C}_{r s}\right| r\right\rangle_{u}^{\prime}{ }_{g}^{\prime}\left\langle s\left|+\widetilde{D}_{r s}\right| r\right\rangle_{u}^{\prime}{ }_{u}^{\prime}\langle s|\right]
$$

where $\widetilde{A}_{r s}, \widetilde{B}_{r s}, \widetilde{C}_{r s}$ and $\widetilde{D}_{r s}$ are constants which can be computed from (4.9), (4.12). The first term on the right hand side of (5.4) describes the contribution from renormalized physical states whereas the other terms represent contribution from renormalized unphysical and pure gauge states.

\footnotetext{
${ }^{10}$ This generalizes the result of [44] in the absence of mass renormalization.
} 
Let us now examine the residues at the poles in (5.3) at leading order in string perturbation theory. First consider the residue at a physical pole. This is given by the products of the components of $\bar{\Gamma}_{1}$ and $\bar{\Gamma}_{2}$ along the corresponding physical state $|\alpha\rangle_{p}^{\prime}$. At the tree level the relevant component of $\bar{\Gamma}_{1}$ is given by the contribution to the full $(m+1)$ point tree amplitude with external states $|\alpha\rangle_{p}^{\prime}$ and $m$ other massless / BPS / special states, and similarly the relevant component of $\bar{\Gamma}_{2}$ is given by the contribution to the full $(n+1)$ point tree amplitude with external states $|\alpha\rangle_{p}^{\prime}$ and $n$ other massless / BPS / special states. Since in the leading order $|\alpha\rangle_{p}^{\prime}$ is given by a linear combination of tree level physical state $|\alpha\rangle_{p}$ and a pure gauge state, and since the pure gauge states decouple in the on-shell tree level amplitude, we can replace $|\alpha\rangle_{p}^{\prime}$ by $|\alpha\rangle_{p}$ in computing the leading order contribution to the relevant components of $\bar{\Gamma}_{1}$ and $\bar{\Gamma}_{2}$. Thus in the leading order the residue at the physical pole is given by the product of two tree level S-matrix elements - one with $(m+1)$ external states and the other one with $(n+1)$ external states. As long as these are non-zero, the residue at the corresponding physical pole will be non-zero. Higher order contributions can correct the residue but cannot make this vanish in perturbation theory. Thus even after including higher order corrections, the corresponding physical mass ${ }^{2}$ 's will appear as the locations of the poles in the $-k^{2}$ plane of the original S-matrix element involving $(m+n)$ external massless / BPS / special states.

Let us now turn to the contribution from the unphysical / pure gauge states. It follows from (4.9), (4.15), (4.17) and (5.4) that for $k^{2}=-m^{2}$ and leading order in $\lambda$, the coefficients $\widetilde{D}_{r s}$ vanish. On the other hand the same equations show that in this approximation $|s\rangle_{g}^{\prime}=|s\rangle_{g}$. Thus the residue is given by a sum of products of appropriate components of $\bar{\Gamma}_{1}$ and $\bar{\Gamma}_{2}$, and in each of these terms either the component of $\bar{\Gamma}_{1}$ or the component of $\bar{\Gamma}_{2}$ (or both) is aligned along a tree level pure gauge state $|s\rangle_{g}$. Thus this factor is given by a tree level amplitude, one of whose external states is $|s\rangle_{g}$ and the other states are on-shell massless / pure gauge / special states. Since this vanishes due to BRST invariance, we conclude that at least at leading order in $\lambda$ the unphysical states do not contribute to the poles in the S-matrix elements of massless / BPS / special states.

Before concluding this section we would like to note that the various quantities which appear in (5.3) - e.g. $\bar{\Gamma}, \bar{\Gamma}_{1}^{T} \mathcal{I} P_{T}, P_{T} \bar{\Gamma}_{2}$ etc. — can be defined without having to explicitly identify the 1PI Riemann surfaces by following the same strategy as in section 4.2. For example we have the relations

$$
\begin{aligned}
\bar{\Gamma}_{1}^{T} \mathcal{I} P_{T} & =\Gamma_{1}^{T} \mathcal{I}\left(1-\left(k^{2}+m^{2}\right)^{-1} \mathcal{I} \widetilde{F}_{T}\right) P_{T}, \\
P_{T} \bar{\Gamma}_{2} & =\left(1-\left(k^{2}+m^{2}\right)^{-1} \mathcal{I} \widetilde{F}_{T}\right) P_{T} \Gamma_{2}, \\
\bar{\Gamma} & =\Gamma-\Gamma_{1}^{T} \mathcal{I}\left(k^{2}+m^{2}\right)^{-1}\left(1-\left(k^{2}+m^{2}\right)^{-1} \mathcal{I} \widetilde{F}_{T}\right) P_{T} \Gamma_{2} .
\end{aligned}
$$

Since $\Gamma, \Gamma_{1}$ and $\Gamma_{2}$ are full amplitudes, their definitions do not require us to divide the moduli space of Riemann surfaces into $1 \mathrm{PI}$ and $1 \mathrm{PR}$ parts. The definition of $\widetilde{F}_{T}$ given in section 4.2 also does not require this division. Thus $\bar{\Gamma}_{1}^{T} \mathcal{I} P_{T}, P_{T} \bar{\Gamma}_{2}$ and $\bar{\Gamma}$ defined via (5.5) also do not require this divison. This observation will be useful when we generalize the analysis to super and heterotic string theories. 


\section{All order results}

We shall now combine the results of section 4.5 and section 5 to prove some all order results in a generic situation. For this we need to first explain what we mean by a generic situation. The conditions under which our arguments will hold are listed below.

1. We assume that the degeneracies between physical and unphysical masses are lifted at first order in perturbation theory. Otherwise our prescription of section 4 of computing renormalized physical masses will have to be modified.

2. We have seen that to leading order the residue at a particular physical mass ${ }^{2}$ of an S-matrix element of external massless / BPS / special states is proportional to the product of S-matrix elements of two lower point tree level S-matrix elements each of which contains, as one of the external states, the physical state whose mass we are interested in. We shall assume that it is possible to choose the external massless / BPS / special states of the original amplitude in such a way that both these lower point S-matrix elements are non-vanishing at tree level. Had we restricted the external states to be only massless or BPS states then this fails in some cases, as was illustrated in [32]. (A particular example of this is the $\mathrm{SO}(32)$ spinor states of ten dimensional $\mathrm{SO}(32)$ heterotic string theory; these cannot appear as one particle intermediate states in the scattering of massless external states which are all in the adjoint or singlet representation of $\mathrm{SO}(32)$.) However at present we do not know of an example where it fails even after we allow as external states the special states introduced in [32]. Once the residue at the pole can be made non-vanishing at leading order, higher order corrections can modify the residue but cannot make it vanish in perturbation theory.

3. We have seen in section 4.5 that the renormalized masses of unphysical / pure gauge states do in general depend on the choice of local coordinates. We shall assume that this is true in all cases, 1.e. there is no renormalized mass corresponding to unphysical / pure gauge states which is accidentally independent of the choice of local coordinates.

Next we shall combine the genericity assumption with some of the relevant results in section 4.5, section 5 and ref. [32] to draw the following conclusions:

1. In a generic situation the renormalized masses of the unphysical / pure gauge states depend on the choice of local coordinates.

2. It was shown in section 4.5 the renormalized masses of physical states do not depend on the choice of local coordinates at least to order $\lambda$.

3. In a generic situation the mass ${ }^{2}$ of physical states appear as poles in the $-k^{2}$ plane of some S-matrix of massless / BPS / special states.

4. It was also shown near the end of section 5 that the unphysical / pure gauge states do not contribute poles in the S-matrix of massless / BPS / special states at least to leading order in $\lambda$. 
5. The S-matrix involving external massless / BPS / special states do not depend on the choice of local coordinates to all orders in $\lambda[32]$.

Let us now combine these results. Points 1 and 5 show, to all orders in $\lambda$, that the unphysical / pure gauge states cannot appear as intermediate states in the S-matrix of massless / BPS / special states. This is consistent with the leading order result mentioned in point 4 . On the other hand points 3 and 5 show, to all orders in $\lambda$, that the mass ${ }^{2}$ of physical states cannot depend on the choice of local coordinates. This is consistent with the leading order result described in point 2.

We can also extend this argument to prove the invariance of the S-matrix elements of general external physical states under a change of local coordinates. For this we note that as long as each of the external states have non-zero tree level amplitude with some set of massless / BPS / special states, we can replace each of the massive, non-BPS and non-special external physical states by the corresponding combination of massless / BPS / special states and examine the corresponding S-matrix for values of momenta where the intermediate physical states of interest go on-shell. The desired S-matrix can then be found by examining the residue at the pole. ${ }^{11}$ Since the S-matrix of massless / BPS / special states is invariant under a change in the local coordinate system, its residues at various poles must also be invariant under a change of local coordinates. This establishes the invariance of the S-matrix elements involving general external physical states under a change of the local coordinate system.

\section{Generalizations to heterotic and super string theories}

We shall now briefly discuss generalizations to heterotic and superstring theories. We shall restrict our discussion to the Neveu-Schwarz (NS) sector, and work with picture number -1 states. In this case the discussion of section 3 can be adapted with few changes:

1. The discussion in section 3.1 remains valid without any change.

2. In the analysis of section 3.2 we need to choose local superconformal coordinate system $(w, \xi)$ around every puncture for defining off-shell amplitudes, and $f_{i} \circ V_{i}$ will label the transform of the vertex operator $V_{i}$ by the superconformal transformation $f_{i}$ that relates the local coordinates near the $i$-th puncture to the reference superconformal coordinates on the super Riemann surface. The detailed analysis of the integration measure (ghost insertions) for off-shell amplitudes can be carried out by combining the description of the measure for on-shell amplitudes in super and heterotic string theories given in [1] with the description of the measure for off-shell amplitudes in bosonic string theory given in [33, 34].

3. The gluing of two Riemann surfaces is implemented via the identification [1]

$$
w_{1} w_{2}=q_{N S}, \quad w_{2} \xi_{1}=\varepsilon \xi_{2}, \quad w_{1} \xi_{2}=-\varepsilon \xi_{1}, \quad \xi_{1} \xi_{2}=0, \quad \varepsilon= \pm \sqrt{-q_{N S}},
$$

\footnotetext{
${ }^{11}$ If the physical state under consideration is unstable then this is the only way to define its 'S-matrix' since the state does not exist as asymptotic state.
} 
and we need to sum over both choices of the sign of $\varepsilon$, leading to GSO projection.

4. The choice of local superconformal coordinates should be compatible with gluing in the same way as in the case of bosonic string theory. We shall also require that the contours in the supermoduli space over which we integrate [1] should be compatible with gluing. In particular this means that in situations where we can integrate out the odd moduli at the expense of inserting picture changing operators $[45,46]$, the locations of the picture changing operators on the glued Riemann surface should be those induced from the locations of the picture changing operators on the lower genus Riemann surfaces which are being glued. A consistent super or heterotic string field theory should automatically satisfy this property in a Siegel like gauge. Construction of classical superstring field theory satisfying these requirements can be found in $[47,48]$.

5. We can now define $\widetilde{F}_{T}$ following the procedure outlined in section 4.2. This avoids having to divide the super Riemann surfaces into 1PI and 1PR surfaces, and directly gives us the expression for $\widetilde{F}_{T}$ in terms of the full off-shell two point function $F_{T}$ of mass level $m$ states. Similarly generalization of the analysis of section 5 can also be carried out by defining $\bar{\Gamma}, \bar{\Gamma}_{1}^{T} \mathcal{I} P_{T}, P_{T} \bar{\Gamma}_{2}$ etc. as in (5.5) instead of in terms of $1 \mathrm{PI}$ super Riemann surfaces.

The rest of the analysis can be carried out in a straightforward matter and we arrive at the same conclusions as in the case of bosonic string theory.

The difficulty in the Ramond sector stems from the fact that there is no natural inner product between states in the $-1 / 2$ picture since the inner product pairs states in the $-1 / 2$ picture to states in the $-3 / 2$ picture. Thus generalization of the analysis of section 3.1 will require us to work with picture number $-1 / 2$ and $-3 / 2$ states together. On the other hand superstring perturbation theory naturally uses $-1 / 2$ picture vertex operators [1]. This seems to be a technical issue which needs to be addressed, possibly by introducing a $\delta\left(\gamma_{0}\right)$ in the definition of the inner product (3.1) so as to get a non-vanishing inner product between two picture number $-1 / 2$ states. We hope to return to this issue in the future.

\section{Acknowledgments}

We thank Rajesh Gopakumar, Michael Green and Edward Witten for useful discussions, and Barton Zwiebach for useful discussions and critical comments on an earlier version of the manuscript. We would like to thank IIT, Kharagpur and the National Strings Meeting held there for hospitality during the course of of this work. A.R. would like to thank HRI, Allahabad for hospitality during the initial and final stages of this work. A.S. would like to thank the organizers of the National Strings Meeting in IIT Kharagpur, MathPhys 2014 conference at Rykkyo University and Asian Winter School at Puri, where preliminay version of this work was presented. The work of R.P. and A.S. was supported in part by the DAE project 12-R\&D-HRI-5.02-0303. A.R. was supported by the Ramanujan studentship of Trinity College, Cambridge, and his research leading to these results has also received funding from the European Research Council under the European Community's Seventh 
Framework Programme (FP7/2007-2013) / ERC grant agreement no. [247252]. The work of A.S. was also supported in part by the J. C. Bose fellowship of the Department of Science and Technology, India.

Open Access. This article is distributed under the terms of the Creative Commons Attribution License (CC-BY 4.0), which permits any use, distribution and reproduction in any medium, provided the original author(s) and source are credited.

\section{References}

[1] E. Witten, Superstring Perturbation Theory Revisited, arXiv:1209.5461 [INSPIRE].

[2] A. Belopolsky, De Rham cohomology of the supermanifolds and superstring BRST cohomology, Phys. Lett. B 403 (1997) 47 [hep-th/9609220] [INSPIRE].

[3] A. Belopolsky, New geometrical approach to superstrings, hep-th/9703183 [INSPIRE].

[4] A. Belopolsky, Picture changing operators in supergeometry and superstring theory, hep-th/9706033 [INSPIRE].

[5] E. D'Hoker and D.H. Phong, Two loop superstrings. I. Main formulas, Phys. Lett. B 529 (2002) 241 [hep-th/0110247] [INSPIRE].

[6] E. D'Hoker and D.H. Phong, Two loop superstrings. II. The Chiral measure on moduli space, Nucl. Phys. B 636 (2002) 3 [hep-th/0110283] [InSPIRE].

[7] E. D'Hoker and D.H. Phong, Two loop superstrings. III. Slice independence and absence of ambiguities, Nucl. Phys. B 636 (2002) 61 [hep-th/0111016] [INSPIRE].

[8] E. D'Hoker and D.H. Phong, Two loop superstrings IV: The Cosmological constant and modular forms, Nucl. Phys. B 639 (2002) 129 [hep-th/0111040] [INSPIRE].

[9] E. D'Hoker and D.H. Phong, Two-loop superstrings. V. Gauge slice independence of the N-point function, Nucl. Phys. B 715 (2005) 91 [hep-th/0501196] [INSPIRE].

[10] E. D'Hoker and D.H. Phong, Two-loop superstrings VI: Non-renormalization theorems and the 4-point function, Nucl. Phys. B 715 (2005) 3 [hep-th/0501197] [INSPIRE].

[11] E. D'Hoker and D.H. Phong, Two-Loop Superstrings. VII. Cohomology of Chiral Amplitudes, Nucl. Phys. B 804 (2008) 421 [arXiv:0711.4314] [inSPIRE].

[12] E. Witten, Notes On Supermanifolds and Integration, arXiv:1209.2199 [INSPIRE].

[13] E. Witten, Notes On Super Riemann Surfaces And Their Moduli, arXiv:1209.2459 [INSPIRE].

[14] E. Witten, More On Superstring Perturbation Theory, arXiv:1304.2832 [INSPIRE].

[15] E. Witten, Notes On Holomorphic String And Superstring Theory Measures Of Low Genus, arXiv:1306.3621 [INSPIRE].

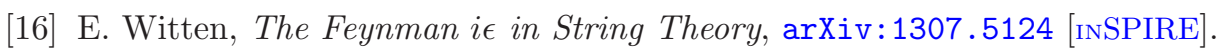

[17] R. Donagi and E. Witten, Supermoduli Space Is Not Projected, arXiv:1304.7798 [INSPIRE].

[18] S. Weinberg, Radiative corrections in string theory, in The Oregon Meeting, Proceedings of the Annual Meeting of the Division of Particles and Fields of the APS, Eugene, Oregon, 1985, edited by R.C. Hwa, World Scientific, Singapore (1986). 
[19] N. Seiberg, Anomalous Dimensions and Mass Renormalization in String Theory, Phys. Lett. B 187 (1987) 56 [inSPIRE].

[20] H. Ooguri and N. Sakai, String Loop Corrections From Fusion of Handles and Vertex Operators, Phys. Lett. B 197 (1987) 109 [INSPIRE].

[21] H. Yamamoto, One Loop Mass Shifts in O(32) Open Superstring Theory, Prog. Theor. Phys. 79 (1988) 189 [INSPIRE].

[22] A. Sen, Mass Renormalization and BRST Anomaly in String Theories, Nucl. Phys. B 304 (1988) 403 [inSPIRE].

[23] S.R. Das, Renormalizing Handles and Holes in String Theory, Phys. Rev. D 38 (1988) 3105 [INSPIRE].

[24] S.-J. Rey, Unified View of BRST Anomaly and Its Cancellation in String Amplitudes, Nucl. Phys. B 316 (1989) 197 [InSPIRE].

[25] B. Sundborg, Selfenergies of Massive Strings, Nucl. Phys. B 319 (1989) 415 [InSPIRE].

[26] N. Marcus, Unitarity and Regularized Divergences in String Amplitudes, Phys. Lett. B 219 (1989) 265 [INSPIRE].

[27] K. Amano and A. Tsuchiya, Mass Splittings and the Finiteness Problem of Mass Shifts in the Type II Superstring at One Loop, Phys. Rev. D 39 (1989) 565 [InSPIRE].

[28] C.J.-H. Lee, BRST anomalies and mass renormalization with anomalous U(1) gauge symmetries in string theory, UMI-92-24830.

[29] A. Berera, The Mass renormalization of string theory, Phys. Rev. D 49 (1994) 6674 [INSPIRE].

[30] L. Del Debbio, E. Kerrane and R. Russo, Mass corrections in string theory and lattice field theory, Phys. Rev. D 80 (2009) 025003 [arXiv: 0812.3129] [INSPIRE].

[31] D. Chialva, String Mass Shifts, Nucl. Phys. B 819 (2009) 225 [arXiv:0903.3979] [InSPIRE].

[32] R. Pius, A. Rudra and A. Sen, Mass Renormalization in String Theory: Special States, arXiv: 1311.1257 [INSPIRE].

[33] B. Zwiebach, Closed string field theory: Quantum action and the B-V master equation, Nucl. Phys. B 390 (1993) 33 [hep-th/9206084] [INSPIRE].

[34] P.C. Nelson, Covariant Insertion of General Vertex Operators, Phys. Rev. Lett. 62 (1989) 993 [INSPIRE].

[35] P. Di Vecchia et al., $N$ Point g Loop Vertex for a Free Bosonic Theory With Vacuum Charge Q, Nucl. Phys. B 322 (1989) 317 [InSPIRE].

[36] A.G. Cohen, G.W. Moore, P.C. Nelson and J. Polchinski, An Off-Shell Propagator for String Theory, Nucl. Phys. B 267 (1986) 143 [InSPIRE].

[37] A.G. Cohen, G.W. Moore, P.C. Nelson and J. Polchinski, Semi off-shell string amplitudes, Nucl. Phys. B 281 (1987) 127 [INSPIRE].

[38] L. Álvarez-Gaumé, C. Gomez, G.W. Moore and C. Vafa, Strings in the Operator Formalism, Nucl. Phys. B 303 (1988) 455 [INSPIRE].

[39] L. Álvarez-Gaumé, C. Gomez, P.C. Nelson, G. Sierra and C. Vafa, Fermionic Strings in the Operator Formalism, Nucl. Phys. B 311 (1988) 333 [INSPIRE]. 
[40] H. Hata and B. Zwiebach, Developing the covariant Batalin-Vilkovisky approach to string theory, Annals Phys. 229 (1994) 177 [hep-th/9301097] [INSPIRE].

[41] A. LeClair, M.E. Peskin and C.R. Preitschopf, String Field Theory on the Conformal Plane. 1. Kinematical Principles, Nucl. Phys. B 317 (1989) 411 [INSPIRE].

[42] A. LeClair, M.E. Peskin and C.R. Preitschopf, String Field Theory on the Conformal Plane. 2. Generalized Gluing, Nucl. Phys. B 317 (1989) 464 [INSPIRE].

[43] L. Rastelli and B. Zwiebach, The Off-shell Veneziano amplitude in Schnabl gauge, JHEP 01 (2008) 018 [arXiv:0708.2591] [INSPIRE].

[44] J. Polchinski, Factorization of Bosonic String Amplitudes, Nucl. Phys. B 307 (1988) 61 [INSPIRE].

[45] D. Friedan, E.J. Martinec and S.H. Shenker, Conformal Invariance, Supersymmetry and String Theory, Nucl. Phys. B 271 (1986) 93 [INSPIRE].

[46] E.P. Verlinde and H.L. Verlinde, Multiloop Calculations in Covariant Superstring Theory, Phys. Lett. B 192 (1987) 95 [inSPIRE].

[47] R. Saroja and A. Sen, Picture changing operators in closed fermionic string field theory, Phys. Lett. B 286 (1992) 256 [hep-th/9202087] [INSPIRE].

[48] N. Berkovits, Y. Okawa and B. Zwiebach, WZW-like action for heterotic string field theory, JHEP 11 (2004) 038 [hep-th/0409018] [INSPIRE]. 